\title{
Differences in adaptive learning across the lifespan are driven by satisficing
}

\author{
Rasmus Bruckner ${ }^{1,2,3, *}$, Matthew R. Nassar ${ }^{4,5}$, Shu-Chen Li ${ }^{6,7}$, Ben Eppinger ${ }^{6,8}$
}

\begin{abstract}
${ }^{1}$ Biological Psychology and Cognitive Neuroscience, Center for Cognitive Neuroscience Berlin, Department of Education and Psychology, Freie Universität Berlin, Berlin, Germany; ${ }^{2}$ Center for Lifespan Psychology, Max Planck Institute for Human Development, Berlin, Germany; ${ }^{3}$ Max Planck School of Cognition, Leipzig, Germany; ${ }^{4}$ Robert J. \& Nancy D. Carney Institute for Brain Science, Brown University, Providence, United States; ${ }^{5}$ Department of Neuroscience, Brown University, Providence, United States; ${ }^{6}$ Lifespan Developmental Neuroscience, Faculty of Psychology, Technische Universität Dresden, Germany; ${ }^{7}$ Centre for Tactile Internet with Human-in-the-Loop, Technische Universität Dresden, Germany; ${ }^{8}$ Department of Psychology and PERFORM Centre, Concordia University, Montreal, Canada; *Corresponding author: Rasmus Bruckner (rasmusb@zedat.fu-berlin.de)
\end{abstract}

\begin{abstract}
Learning is pervasive across the human lifespan and essential for adaptive behavior. Children and older adults are often slower to learn cognitive tasks than young adults. Here we build on established theory formalizing learning as predictive inference and consider the possibility that age-related learning differences emerge from satisficing in this prediction problem - that is, accepting predictions that achieve some sufficient level of accuracy. In our computational formalization of this idea, beliefs are updated through an active process until reaching some criterion value of acceptability, which is set more liberally in children and older adults. In line with our model's predictions, children and older adults showed frequent perseveration when asked to update beliefs from their previous values but were also more highly influenced by environmental manipulations of the starting value from which beliefs are updated. Our model and results provide a unifying perspective on seemingly contradictory findings showing developmental differences in the reliance on internal and external behavioral biases by suggesting that they both emerge from a liberal satisficing policy.
\end{abstract}

\section{Introduction}

Many of our decisions are guided by uncertain information that is not directly observable. For example, we can not directly perceive the quality of a new ice-cream parlor but have to learn about it by sampling different sorts of ice cream (Bolenz et al., 2017). While doing so, smaller differences in the experienced quality (small prediction errors) can be written off as expected daily fluctuations that should not fundamentally change our minds about the parlor's quality. However, unexpectedly large deviations from our beliefs (large prediction errors) could indicate that the quality has systematically changed, for example, because the ice-cream parlor has a new owner. In this case, it may be necessary to update our beliefs more dramatically and consider a different ice-cream parlor. Such attributions of prediction errors to variability versus changepoints can be difficult in the face of uncertainty but are critical for controlling the degree to which new experiences influence learning.

Previous work has identified normative mechanisms derived from principles of optimal Bayesian inference for implementing this sort of belief updating (Behrens et al., 2007; Meyniel and Dehaene, 2017; 


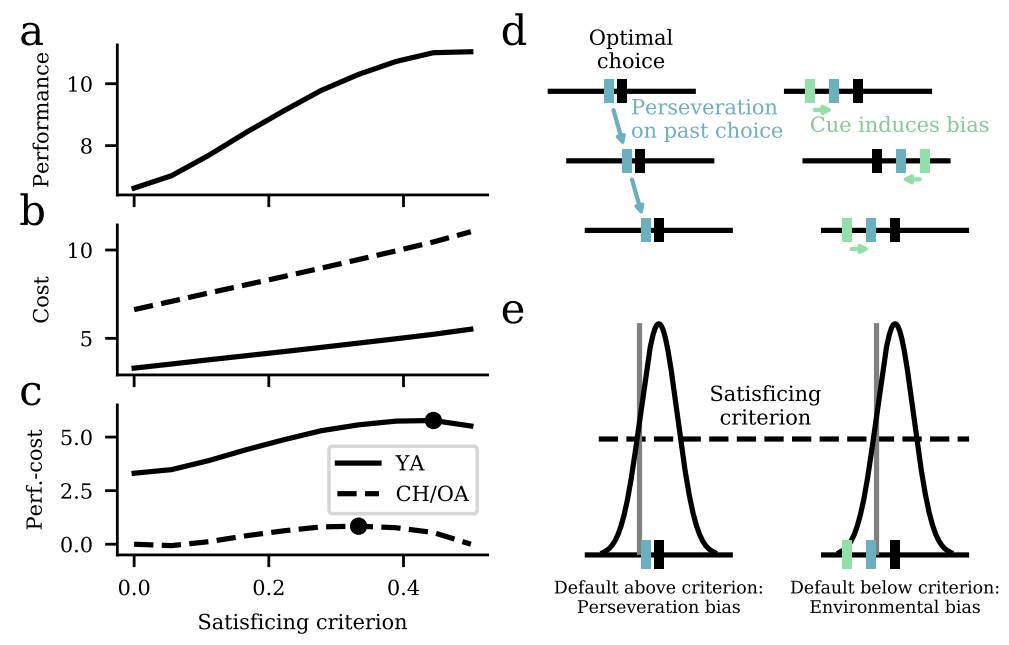

Figure 1. Satisficing. Optimal learning strategies are often associated with high cognitive demands that may exceed limited resources. Therefore, humans might resort to simpler strategies such as the satisficing heuristic, in which they make choices that are sufficiently accurate but not necessarily optimal. a-c) Potential performance, costs, and the trade-off between them across multiple satisficing criteria. a) A low satisficing criterion is associated with the acceptance of almost any choice. However, it also leads to lower learning performance. A higher satisficing criterion means that fewer choices are accepted and therefore higher performance levels. b) At the same time, relying on a satisficing policy with a low criterion is cognitively less demanding than a strategy with a high satisficing criterion. Given cognitive limitations, the subjective costs of learning might vary across age groups, i.e., in children and older adults, learning is more costly compared to younger adults. c) In effect, the costs required to reach an optimal performance level can even exceed the prospective performance. Under those circumstances, satisficing can be an efficient strategy, particularly for children $(\mathrm{CH})$ and older adults (OA). Here, costs are subtracted from performance, showing that particularly children and older adults would reach a higher cost-corrected performance under a more liberal satisficing policy. d) Satisficing might explain two seemingly opposing effects frequently found in children and older adults. On the one hand, they often perseverate on previous choices instead of selecting the optimal option. On the other hand, both age groups show stronger biases through external information such as task cues, often referred to as environmental control. e) In environments where beliefs are updated from previous values, perseveration occurs when the considered choice is above the satisficing criterion and therefore sufficiently accurate. In contrast, environmental cues that determine the initial value from which beliefs are updated lead to environmental control of learning.

Nassar et al., 2010; O’Reilly et al., 2013; Payzan-LeNestour et al., 2013). However, deviations from optimal behavior hint that people might rely on computationally cheaper alternatives in cases where they achieve satisfactory predictions. Many realistic learning and decision-making problems are complex and have to be solved under limited time so that optimal solutions are unfeasible. Therefore, humans might use heuristic strategies that demand less cognitive resources while ensuring a reasonable level of performance. One such heuristic is satisficing, which aims for choices that meet an acceptable criterion that can be efficiently achieved based on incomplete information or simpler computations than required for optimal solutions (Gigerenzer and Gaissmaier, 2011; Simon, 1955, 1956).

Children and older adults often underperform relative to young adults across a wide range of cognitive tasks (Chowdhury et al., 2013; De Boer et al., 2017; Eppinger et al., 2008, 2009, 2013; Hämmerer et al., 2011, 2019; Nassar et al., 2016; Rutledge et al., 2009; Samanez-Larkin et al., 2010, 2012, 2014; Van den Bos et al., 2012; Van der Schaaf et al., 2011). Some studies suggest that these age groups are more reliant on internally generated biases leading to perseveration (Blackwell and Munakata, 2014; Blackwell et al., 2014; Carroll et al., 2016; Cepeda and Munakata, 2007; Crone et al., 2004a,b; Head et al., 2009; Munakata et al., 2012; Ridderinkhof et al., 2002; Rutledge et al., 2009), whereas others come to the opposite conclusion and pin the difference on an over-reliance on available external cues (Alarcón and Bonardi, 2020; Chatham et al., 2009; Craik and Bialystok, 2006; Gonthier et al., 2019; Lindenberger and Mayr, 2014; Munakata 
et al., 2012; Troller-Renfree et al., 2020). These findings have previously mainly been interpreted as learning and decision-making deficits but an alternative perspective is that children and older adults show more satisficing behavior, potentially to adapt to age-related changes in available cognitive resources.

Both biases to perseverate on previous behavior and to rely more strongly on environmental information might emerge from a low satisficing criterion that leads children and older adults to accept a considered choice more swiftly. Figure 1a-c shows how the costs and benefits of learning behavior could affect the satisficing criterion. We assume that belief updating is a dynamic process where the plausibility of the candidate choice is sequentially evaluated until reaching a satisficing criterion at which an acceptable choice is established. Each moment-to-moment evaluation of the current belief is associated with cognitive costs. A low satisficing criterion requires fewer evaluations but would be associated with lower accuracy of the updated belief. With higher demands on their limited cognitive capacities to engage in such momentto-moment evaluations, children and older adults may set a lower satisficing criterion that would make them accept choices that seem plausible but are not necessarily optimal. Accordingly, when updating from a previous belief, perseverative behavior occurs because individuals perceive their previous choice as sufficiently accurate (Fig. 1d, left). In this case, the last choice is above the satisficing criterion and therefore directly accepted (Fig. 1e, left). In contrast, in the presence of external cues that determine the starting point of the updating process, individuals sequentially consider several choice options but, depending on their satisficing criterion, terminate the choice process as soon as they identify a satisfying option (Fig. 1d,e right). In effect, the reported belief update is biased towards the externally determined initial value.

In the present study, we examined whether satisficing accounts for age-related learning differences using a predictive-inference task and computational modeling. In the first experiment, where beliefs were updated directly from their previous values, performance differences between age groups were primarily related to more perseverative responses in children and older adults than in adolescents and younger adults. In a second experiment, we manipulated the starting position for participant predictions. Consistent with the predictions of a satisficing model, this manipulation impaired the performance of children and older adults even further. Together, the results suggest that stronger internal and external behavioral biases in children and older adults than in younger adults arise from a more liberal satisficing policy. Our model provides a mechanistic interpretation of our results, a parsimonious explanation for several seemingly contradictory results in the developmental and aging literature, and highlights how decisionmaking strategies can lead to different task-dependent biases across the lifespan.

\section{Results}

\section{Task design and model predictions}

To examine age differences in adaptive learning across the lifespan, we analyzed experimental data of 33 children ( 8 to 10 years, median age 9 years), 29 adolescents (13 to 17 years, median age 15 years), 32 younger adults (20 to 28 years, median age 24 years), and 35 older adults (62 to 80 years, median age 71 years). In the experimental task, participants were instructed to maximize the number of collected coins that were contained in bags dropped by an invisible helicopter (Nassar et al., 2016) (Fig. 2a). Participants tried to catch the dropped bags by placing a virtual bucket at the next expected bag location. However, because clouds covered the sky, participants could not directly observe the helicopter but had to learn its location based on the history of observed bags. The environment was windy, which distorted the position of the dropped bags and induced variability in the outcomes. Hence, participants could utilize the bags to estimate the hidden location of the helicopter, but estimates were uncertain. Technically, we generated these outcomes using a Gaussian distribution, where the mean was equal to the position of the hidden helicopter and the standard deviation led to variability across outcomes. Moreover, the helicopter 
a

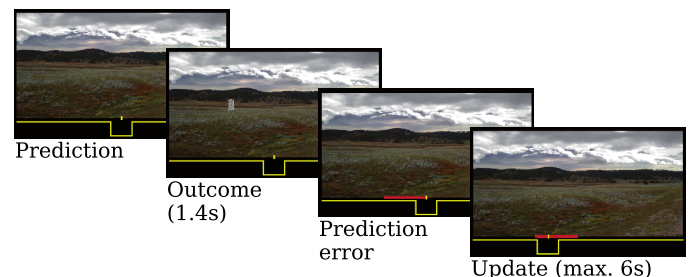

$\mathrm{b}$
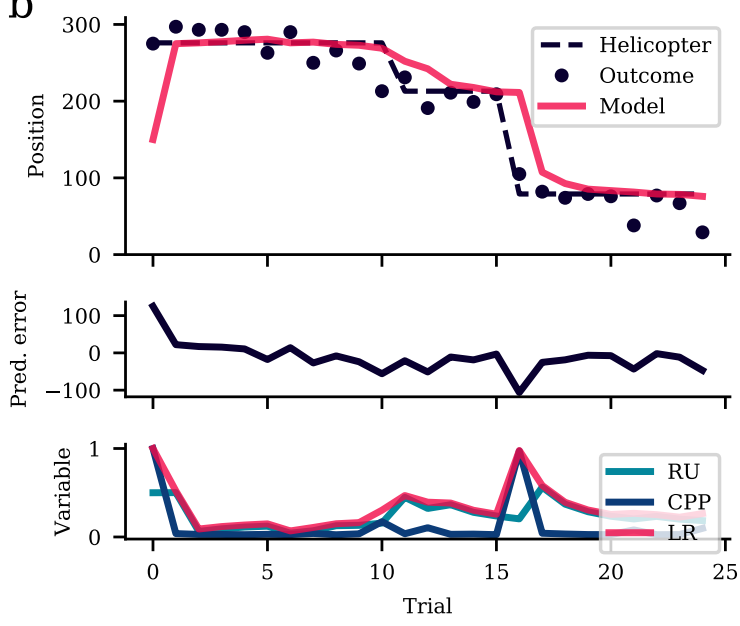

Figure 2. Experimental task and reduced Bayesian model. Experimental task and reduced Bayesian model. In the experimental task, participants tried to catch bags containing gold or stone that were dropped by a hidden helicopter. Participants caught the bags in a bucket placed at the expected position of the bag on the screen (Prediction). The helicopter then dropped the bag (Outcome), a red line marked the prediction error (Prediction error), and participants could adjust their prediction of the bag location for the next trial (Update). b) The helicopter position (dashed line, first panel) was stable on most trials and only changed occasionally (changepoint). Outcomes (black dots, first panel) were noisy because they were randomly displaced with respect to the helicopter position. The red line in the first panel shows the prediction of the reduced Bayesian model that inferred the position of the hidden helicopter based on the position of the dropped bags. To update its predictions, the model computed prediction errors (second panel). The learning rate (LR; third panel) controls the influence of prediction errors on the model's belief update. In the reduced Bayesian model, the magnitude of the learning rate depends on changepoint probability (CPP) and relative uncertainty (RU).

occasionally changed its position, which we refer to as changepoint. After a changepoint, previously learned predictions were inaccurate, and the best strategy was to re-learn the hidden helicopter location. Notably, because the helicopter was not directly observable, it could be difficult to distinguish changepoints from variability due to the wind in the environment. Participants received rewards for catching gold coins that emerged from dropped bags on a random subset of trials (see Experimental task).

Learning behavior in this task can be formally captured by a reduced Bayesian model that adjusts the rate of learning on each trial (Fig. 2b) (McGuire et al., 2014; Nassar et al., 2010, 2012, 2016; Vaghi et al., 2017). The model learns according to principles of an error-correcting delta rule that is often applied in reinforcement-learning studies (Daw, 2014; Dayan and Daw, 2008). In particular, the model learns a belief about the helicopter's trial-wise position based on prediction errors that result from a comparison between actual and predicted outcomes. To revise beliefs appropriately, the model scales prediction errors according to a learning rate to produce an update in the expected helicopter location. The Bayesian model dynamically adjusts the learning rate from trial to trial, reflecting attributions of prediction errors to variability across outcomes versus changepoints in the helicopter location. After smaller prediction errors that are more likely due to outcome variability, the model subtly regulates the learning rate according to its relative uncertainty about the hidden helicopter location. In contrast, prediction errors that are more likely due to a changepoint, as indicated by changepoint probability, lead to higher learning rates and a more substantial belief update (see Reduced Bayesian model).

\section{Age-related learning differences}

To examine age-related performance differences we compared the estimation errors, which reflect the absolute difference between the true but unknown location of the helicopter and the participants' prediction of the next outcome, across age groups (Fig. 3a). Lower values indicate a more accurate estimate of the 

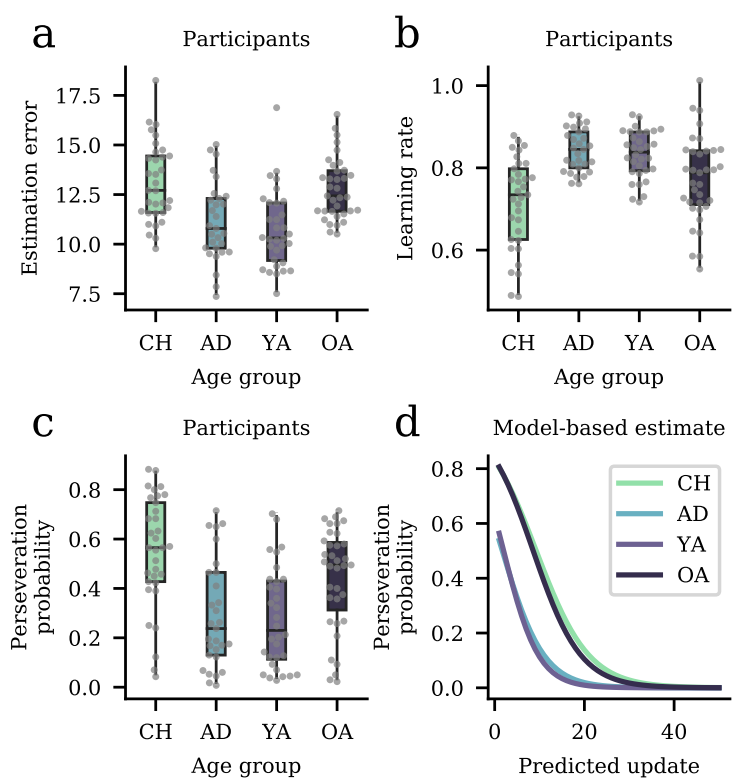

Figure 3. Performance and perseveration analyses. a) Task performance, i.e., estimation errors that indicate the absolute difference between helicopter location and prediction. Lower estimation errors indicate a higher task performance. b) Average learning rates describe the average influence of the prediction errors on the belief updates about the hidden helicopter position. c) Estimated perseveration probability. Higher values indicate more frequent perseveration. d) Model-based perseveration-probability curves as a function of the predicted update of the reduced Bayesian model plotted separately for each age group. The curves indicate that children and older adults were more likely to perseverate, particularly when small and intermediate belief updates were predicted by the reduced Bayesian model.

helicopter location (see Data preprocessing). According to a Kruskal-Wallis one-way analysis of variance on ranks (henceforth referred to as ANOVA), estimation errors were significantly different between the age groups $(H=32.069, p<0.001)$. Post-hoc two-sided Mann-Whitney $\mathrm{U}$ tests indicated that children $($ median $=12.711$, IQR 11.594 to 14.445$)$ showed significantly higher estimation errors compared with adolescents $($ median $=10.791$, IQR 9.794 to $12.304 ; u=724.0, p=0.001$ ) and younger adults $($ median $=$ 10.311, IQR 9.176 to $12.075 ; u=862.0, p<0.001)$. Adolescents $(u=258.0, p=0.001)$ and younger adults $(u=205.0, p<0.001)$ had significantly lower estimation errors compared with older adults $($ median $=$ 12.433, IQR 11.658 to 13.704). The test did not yield significant differences between children and older adults $(u=625.0, p=0.564)$ and between adolescents and younger adults $(u=523.0, p=0.398)$.

To investigate age differences in learning that could have mediated performance differences, we first computed the average learning rates across all trials and compared them between the age groups (Fig. 3b and Average learning-rate analysis). An ANOVA indicated that learning rates were significantly different between the age groups $(H=32.001, p<0.001)$. Post-hoc tests showed significantly lower average learning rates in children (median $=0.734$, IQR 0.626 to 0.798 ) compared with adolescents (median $=0.845$, IQR 0.8 to $0.887 ; u=137.0, p<0.001$ ) and younger adults (median $=0.838$, IQR 0.793 to $0.887 ; u=188.0$, $p<0.001)$. Post-hoc tests also yielded significantly higher learning rates in adolescents compared with older adults (median $=0.788$, IQR 0.711 to $0.842 ; u=738.0, p=0.002$ ) and in younger compared to older adults $(u=779.0, p=0.006)$. The test did not yield significant differences between children and older adults $(u=431.0, p=0.073)$ and between adolescents and younger adults $(u=503.0, p=0.578)$. Thus, children and older adults use lower learning rates than adolescents and younger adults, suggesting that, on average, children and older adults consider prediction errors to a lower degree during belief updating.

To better understand the computational underpinnings of these differences in learning, we analyzed the trial-by-trial dynamics of learning-rate adjustments in the different age groups using the reduced Bayesian model. To do so, we equipped the model with several free parameters that we estimated using a maximum-likelihood approach (see Evaluation and estimation). In line with our previous study comparing younger and older adults in the helicopter task (Nassar et al., 2016), we found some evidence for a reduced consideration of relative uncertainty in older compared to younger adults. However, these differences were unlikely to explain our observed performance differences between the age groups. Moreover, we also 
tested age-related differences in the consideration of changepoint probability and participants' subjective assumption about the frequency of changepoints, which did not indicate significant differences between the age groups (see SI Computational modeling first experiment).

In addition to considering differences in such high-level computations involved in selecting a learning rate, we also considered the possibility that individuals might employ frugal policies for implementing it. For example, our motivating model suggests that satisficing should frequently lead to non-updates, that is, trials where participants perseverate on their previous prediction (Fig. 1). In line with the predictions of the satisficing model, all four age groups showed perseveration in the task, i.e., they repeated the same prediction across two or more consecutive trials (Fig. 3c). An ANOVA yielded significantly different estimated perseveration probabilities across the age groups $(H=27.229, p<0.001)$. The estimated perseveration probability in children (median $=0.565$, IQR 0.428 to 0.748 ) was significantly higher comprared to adolescents (median $=0.238$, IQR 0.13 to $0.465 ; u=752.5, p<0.001$ ), younger adults (median $=0.23$, IQR 0.113 to $0.43 ; u=859.0, p<0.001$ ), and older adults (median $=0.495$, IQR 0.312 to $0.586 ; u=739.5, p=0.047)$. We also found significant perseveration differences between adolescents and older adults $(u=307.0, p=0.007)$ and between younger and older adults $(u=320.0$, $p=0.003)$. The test did not show a significant difference between adolescents and younger adults $(u=$ 478.0, $p=0.845$ ). These results suggest that all age groups perseverated on their predictions, but such perseveration was more pronounced in children and older adults compared to adolescents and younger adults. Taken together, perseveration might indicate a problem - but it could potentially also reflect a strategic decision to minimize updating costs through satisficing (Fig. 1).

We captured these age-related differences in perseveration in our computational model, which allowed for a better explanation of participant data compared to the Bayesian model without perseveration (see SI Model comparison). These model-based analyses revealed that age-related differences in perseveration probability were particularly pronounced in the range of small to intermediate updates (Fig. 3d).

\section{Reducing perseveration leads to more environmental control of learning}

As suggested by the Bayesian model, it could be possible that the high perseveration levels in children and older adults primarily explain their lower task performance. Therefore, in the next step, we empirically tested if perseveration and the associated effects on estimation-error differences between the age groups can be reduced with experimental manipulations. This follow-up experiment contained an effective participant sample of 31 children ( 7 to 11 years, median age 9 years), 25 younger adults (20 to 28 years, median age 24 years), and 34 older adults (61 to 76 years, median age 67 years). Out of efficiency considerations, we did not test adolescents as they showed similar levels of perseveration as younger adults in the first experiment. We used a modified version of the helicopter task that involved two additional experimental manipulations to reduce perseveration. First, we included a reward-magnitude manipulation in which we subdivided trials into high- and low-reward trials, which, however, did not significantly affect the estimated perseveration probability (see SI Results follow-up experiment). Second, we used two different task environments. In the shifting-bucket environment, we randomly perturbed the bucket's initial location at the onset of each trial (Fig. 4a). The consequence of the bucket randomization was that participants could not repeat the previous trial's prediction using a single keypress, as this would now require them to adjust their bucket back to its previous position. We presented this shifting-bucket environment in two out of four task blocks combined with the incentive manipulation.

We next examined the success of the manipulation that shifted the initial bucket location to reduce perseverative behavior. The results revealed that the manipulation strongly reduced the participants' perseveration (Fig. 4c and SI Results follow-up experiment), allowing us to test whether it might rescue task performance in children and older adults. According to an ANOVA, this reduction in perseveration was significantly different between the age groups $(H=18.89, p<0.001)$. The perseveration reduction 
a

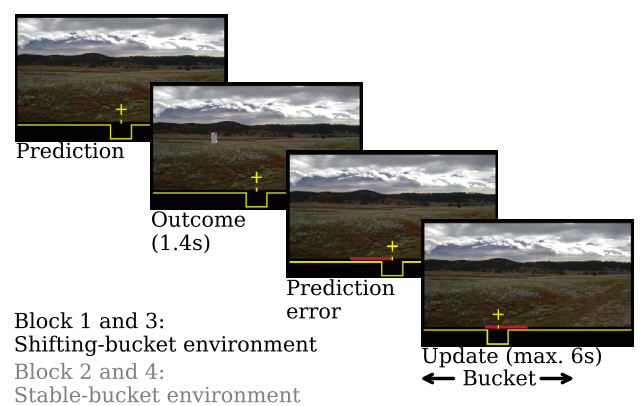

Block 2 and 4 :

Stable-bucket environment
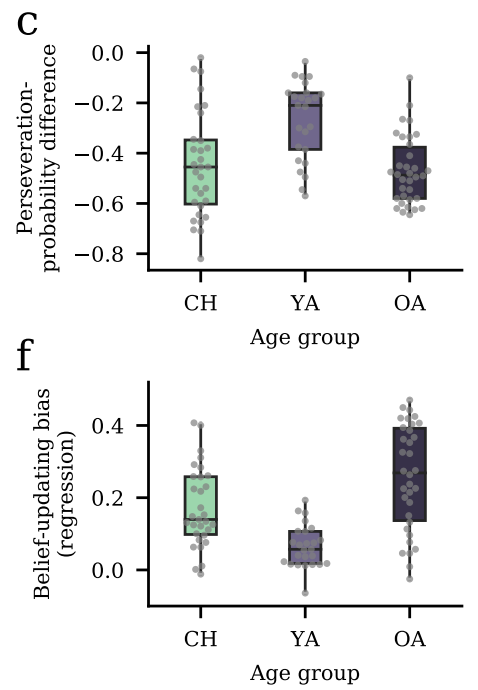

d

g $\mathrm{b}$
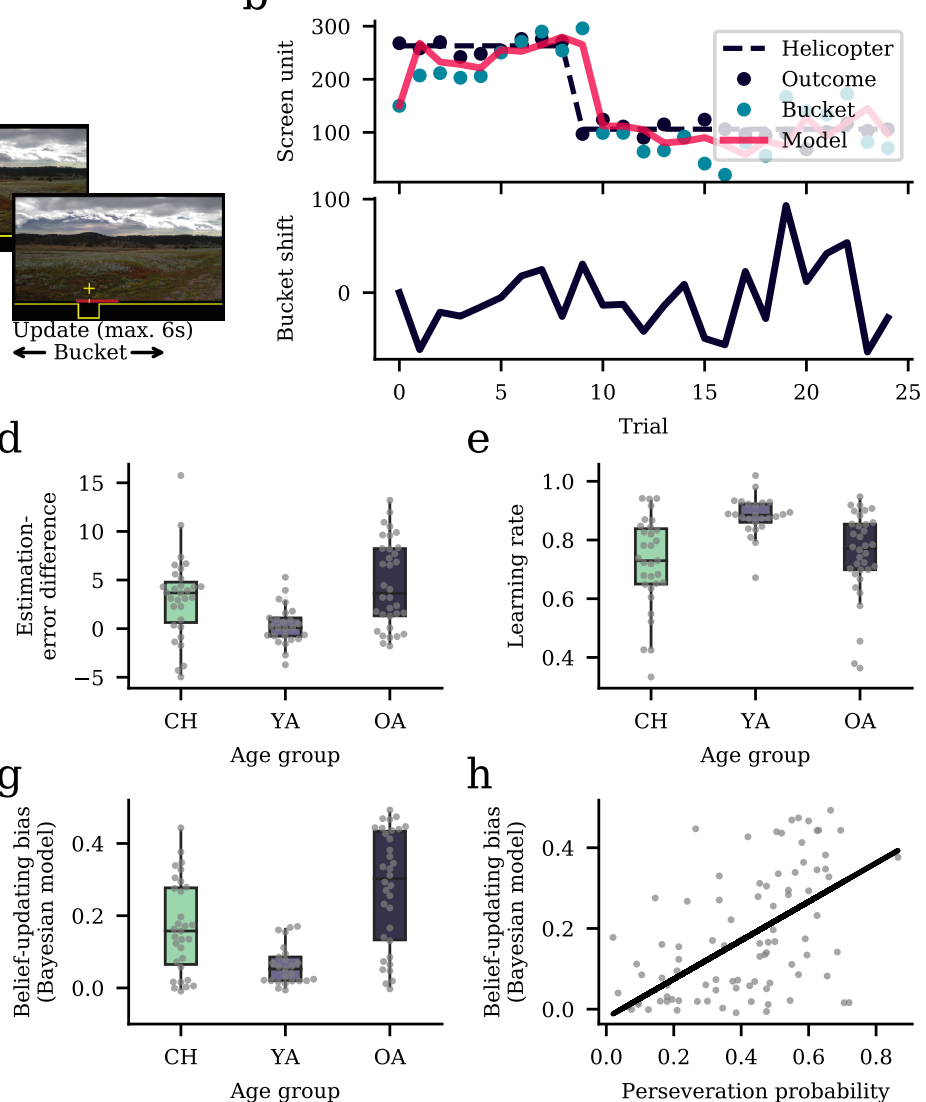

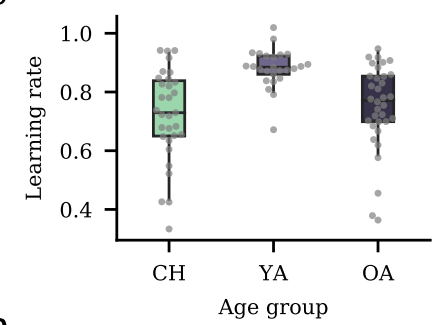

$\mathrm{h}$

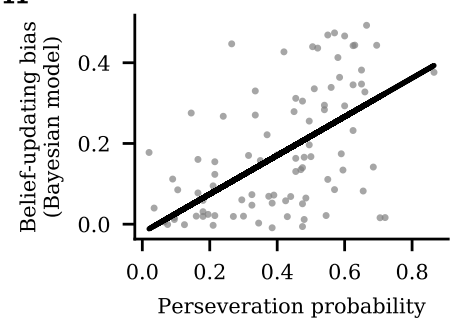

Figure 4. Follow-up experiment with reduced perseveration and additional belief-updating bias. a) In the follow-up experiment, we divided the task into a shifting- and stable-bucket environment. In the shifting-bucket environment, we randomly replaced the bucket's starting location, with which participants tried to catch the bags dropped by the hidden helicopter. In the stable-bucket environment, the bucket stayed at the participants' previous predictions (as in the first experiment). b) The upper plot shows the outcomes in the task (black dots), an example of the predicted outcomes according to a simulation using the reduced Bayesian model (red line) and the starting bucket location (blue dots). To capture a bias of the shifted bucket on belief updating, we added a bias parameter to the model, such that the predictions of the reduced Bayesian model were biased towards the shifted bucket location. The lower panel shows the shift in the initial bucket location, i.e., the difference between the previous and current bucket location. c-d) Difference between the shifting- and stable-bucket environments. c) The negative perseveration-probability difference indicates that perseveration was lower in the shiftingversus stable-bucket environment. d) The positive estimation-error difference indicates that estimation errors were larger in the shifting-bucket environment. e) Empirical estimates of the bucket-bias parameter. f) The estimated bucket-bias parameter and perseveration probability were systematically related in that a higher perseveration probability statistically predicted a stronger belief-updating bias.

in children was significantly higher compared to younger adults $(u=192.5, p=0.001)$ but not compared to older adults $(u=543.5, p=0.834)$. In younger adults, the reduction in perseveration was significantly lower compared to older adults $(u=709.5, p<0.001)$. Lastly, we tested if participants predicted the helicopter at the randomly determined bucket location during some trials, instead of at the location of their last prediction as shown above. However, this analysis revealed that participants hardly ever directly accepted the externally determined location for their prediction (see SI Results follow-up experiment). Thus, our task manipulation successfully normalized the key factor contributing to age-related performance differences in our previous experiment. 
If perseveration were the computational deficit, suppressing perseveration should rescue learning behavior (see SI Model validation). However, according to the predictions of a satisficing model, the performance of children and older adults should be even more impaired on this version of the task (Figure 1e). In line with the satisficing model predictions, the shifting-bucket manipulation did not rescue performance in our target age groups but instead led to additional impairments (Fig. 4d and SI Results follow-up experiment). An ANOVA that tested the within-participant differences in estimation errors between the environments showed a significant difference between the age groups $(H=15.748, p<0.001)$. Post-hoc tests revealed significantly higher estimation-error differences between the bucket-shift environments in children compared to younger adults $(u=577.0, p=0.002)$ and younger adults compared to older adults $(u=186.0$, $p<0.001)$ but not between children and older adults $(u=445.0, p=0.284)$.

We next examined which factors led to these performance decrements in our target groups. Age-related differences in average learning rates failed to provide a reasonable explanation (Fig. 4e and SI Average learning rate follow-up experiment). However, consistent with the predictions of a satisficing model, another possible explanation for the failure of our bucket-shift manipulation to improve performance in children and older adults would be that these groups were misled by the manipulation itself. As reported earlier, it was extremely rare for participants to leave their bucket in the randomly initialized location, but it could be possible that the bucket shift might have a more continuous impact on updated beliefs. With this in mind, we tested if participants were biased by the random shifts in the bucket location (Fig. 4f). An ANOVA revealed that the effect of the randomized initial bucket location on belief updates was significantly different between the age groups $(H=27.289, p<0.001)$. The post-hoc tests showed significantly stronger influences in children (median $=0.14$, IQR 0.098 to 0.258 ) compared to younger adults (median $=0.057$, IQR 0.018 to $0.107 ; u=614.0, p<0.001$ ) and older adults (median $=0.269$, IQR 0.137 to 0.392$)$ compared with younger adults $(u=116.0, p<0.001)$. The effect of the random bucket location on belief updating in children was significantly lower compared older adults $(u=345.0, p=$ 0.017). These results indicate that belief updates in children and older adults were more strongly affected by the externally determined starting location of the bucket compared to younger adults. We confirmed that learning in these age groups was more substantially controlled by the initial bucket location in a model-based analysis, where the model's prediction of the next outcome was biased towards the shifted bucket position (Fig. 4b). This belief-updating bias was governed by an additional free parameter, where higher parameter values indicate a stronger bias that showed similar age-related differences in the effect of the initial location on learning (Fig. $4 \mathrm{~g}$ and SI Computational modeling follow-up experiment).

In summary, the results suggest a relationship between perseverative responses characterizing the behavior of children and older adults in our first experiment, and environmental control of learning that characterizes their behavior in the second. To test whether these two effects might emerge from a common underlying cause, we examined the relationship between the belief-updating bias and perseveration probability (see Robust regression). As would be expected if the two effects emerged from a common cause, participants who tended to perseverate more often in the stable-bucket environment were also more strongly biased by the shift in the bucket location in the shifting-bucket environment (Fig. 4h; regression weight $\left.\beta_{1}=0.4791, z=5.34, p<0.001\right)$. Thus, perseveration in children and older adults was not reflecting a strong adherence to prior beliefs, but rather a sensitivity to the starting value of the belief update, be it internally or externally generated (see also SI Robust regression with age group).

\section{Differences in adaptive learning across the lifespan are driven by satisficing}

The Bayesian model described above does not capture the relationship between internally (perseveration) and externally (environmental control) generated learning biases and thus can not generalize across different task settings. One possible reason for this is that the Bayesian model is agnostic to the initial bucket location and assumes that updates will be entirely made to the most likely location of the helicopter. 
a

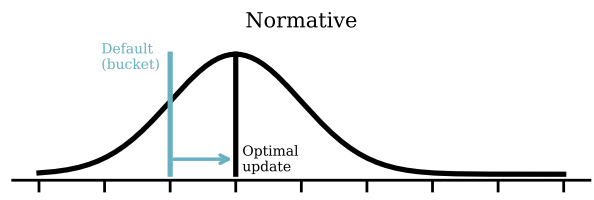

$\mathrm{b}$

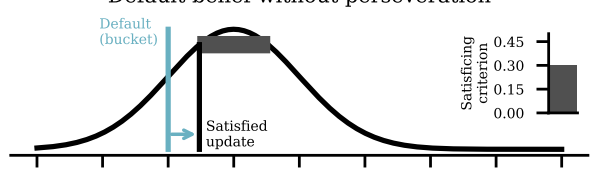

C

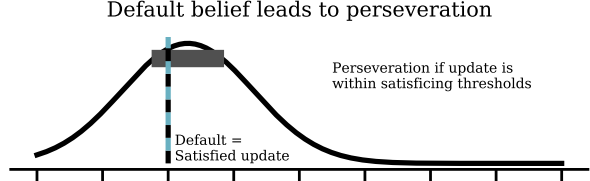

d

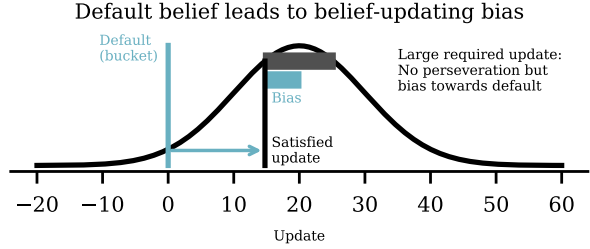

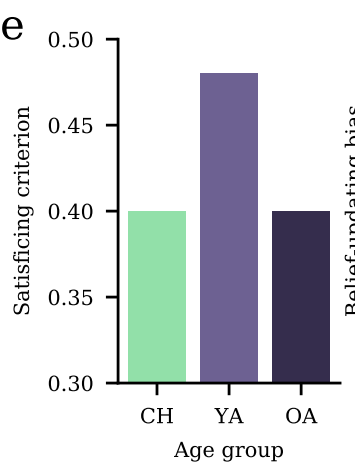

$\mathrm{f}$
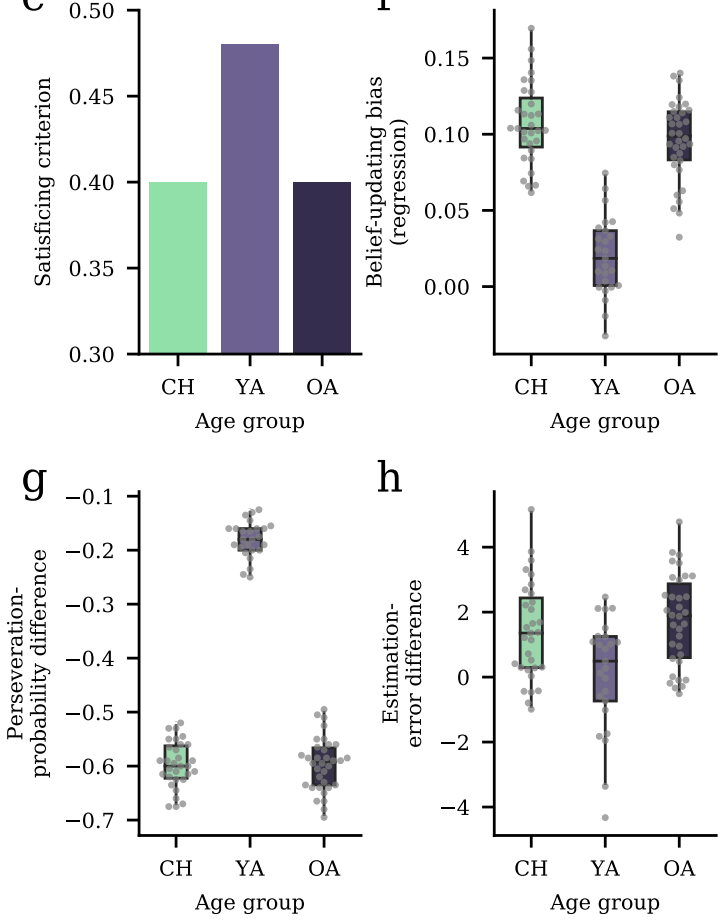

$\mathrm{h}$

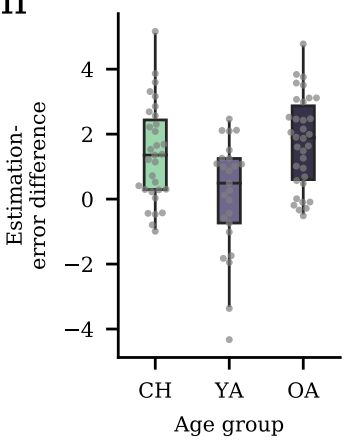

Figure 5. Satisficing model. We formalized the dependency of perseveration and environmental control, which showed that the seemingly contradictory belief-updating behavior in children and older adults could be explained by considering the dynamics of the updating process. a) The normative reduced Bayesian model would always update the bucket to the most likely location of the helicopter. b) The satisficing model updates the bucket position towards the most likely helicopter location until reaching the satisficing criterion, at which an acceptable bucket position is established (here using a satisficing criterion of 0.30). c) If the considered belief is above the satisficing criterion, the model reports the initial belief and perseverates. d) If the current belief is lower than the satisficing criterion, the model updates the bucket until hitting the criterion. This under-adjustment leads to a bias towards the initial belief, i.e., the default bucket location. e) Satisficing criterion during the simulations. f) The simulations reproduce the empirical belief-updating bias (see Fig. 4f), g) the empirical perseveration-probability difference between the shifting- and stable-bucket environment (see Fig. 4c), and h) the estimation-error difference between the environments (see Fig. 4d).

Therefore, we added a satisficing mechanism to our Bayesian model where the initial belief is equal to the starting location of the bucket and subsequently updated in the direction of the most likely helicopter position until reaching some satisficing criterion, at which an acceptable bucket position is established (see Satisficing model). This additional satisficing mechanism captures both of our observed learning biases. As shown in Fig. 5a, the Bayesian model would always update its belief toward the optimal update location. In contrast, the satisficing model updates only if the required update is below a satisficing criterion near the optimal update. In this case, the model keeps updating until the criterion is hit (Fig. 5b). If the required update is above the criterion, the model perseverates because the previous prediction is sufficiently accurate (Fig. 5c). Finally, when the bucket is randomly perturbed, the required update is mostly below the criterion, rendering perseveration unlikely. However, because the model stops the update at the criterion, its reported belief is biased toward the externally determined initial bucket location (Fig. 5d).

We finally tested if the assumed reliance on satisficing might explain the age-related differences that we observed in our experiments. In the following simulations, we assumed a lower satisficing criterion in children and older adults (0.40, indicating the consideration of a broader range of acceptable updates) compared to younger adults (0.48, indicating a narrow range of acceptable updates; Fig. 5e). All other 
parameters were the same for all age groups. The simulated participants showed a comparable pattern of belief-updating biases to the experimental data, where the bias was stronger in children and older adults (Fig. 5f). Moreover, the simulated age groups showed a stronger perseveration-probability difference between the shifting- and stable-bucket environment (Fig. 5g). Lastly, they also showed a similar pattern of estimation-error differences (Fig. 5h). In summary, the satisficing model qualitatively captures the age-related differences in perseveration probability and environmentally controlled belief updating. The model shows that the seemingly contradictory findings across the two experiments could be produced by a common satisficing heuristic that yields different task-dependent learning biases.

\section{Discussion}

This study used a predictive-inference task combined with computational modeling to investigate lifespan age differences in learning under uncertainty. Across two experiments, our findings suggest that age-related differences in adaptive learning are driven by a more liberal satisficing policy in children and older adults. When beliefs are updated from previous values (first experiment), this leads to increased perseveration. In environments with externally generated initial choice options (follow-up experiment), it leads to more substantial environmental control of learning. Therefore, we suggest that these two seemingly contradictory age-related behavioral biases emerge from the same underlying satisficing policy, potentially due to age-related differences in the cognitive capacities available for learning across the lifespan.

Our satisficing model learns the belief about the helicopter location akin to the reduced Bayesian model developed in previous studies (Nassar et al., 2010, 2016). However, in contrast to previous work, it engages in moment-to-moment assessments of the currently considered belief and terminates the updating process when reaching a satisficing criterion. This view of updating as a dynamic process is consistent with a recent theoretical account of model-based decision making by Piray and Daw (2020), who have considered model-based decisions as costly deviations from a default policy. Furthermore, it is in line with a computational account of the anchoring-and-adjustment theory showing that biases induced by cognitive anchors may be rational when faced with limited computational resources (Lieder et al., 2018). Children and older adults were best described as having a lower criterion, producing both more perseveration (compare Fig. 5g to Fig. 4c) and stronger influences of external information on learning (compare Fig. $5 \mathrm{f}$ to Fig. 4f,g) than in their young adult counterparts. While our task allowed us to explicitly manipulate initial beliefs and the differential effects of satisficing, in more standard choice and response tasks, the starting point from which beliefs are updated is not directly observable. In what follows, we provide a unifying framework proposing that satisficing may be a common cause underlying perseverative behavior and greater influences of environmental control in children and older adults across different task domains.

The model is consistent with previous accounts in younger adults that interpret perseveration as a habitual, default response that emerges from repeatedly selecting an option (Gershman, 2020; Miller et al., 2019; Wood and Rünger, 2016). However, it also extends them by highlighting the role of satisficing rather than previous choices per-se. Miller et al. (2019) argued that perseveration could arise from valuefree habitual control of behavior, which competes with goal-directed control on a trial-by-trial level. While this account is consistent with the perseveration results of the first experiment, it can not account for our findings of increased environmental control of learning in the follow-up experiment. Our model assumes that individuals dynamically update the currently considered response until they perceive it as sufficiently accurate. Thus, even within a trial, our model does not assume the arbitration between a habitual and goal-directed system but rather an interplay of the two that depends on the degree of satisficing. Thus, our computational approach may have implications for previous theories of habitual and goaldirected behavior because it provides a mechanism to explain the dynamics of within-trial adjustments of these behavioral strategies (Collins and Cockburn, 2020). Another interpretation of our results is 
that perseveration occurs because the cognitive resources required to represent the action policy exceed cognitive capacities (Gershman, 2020). In our task, the policy determines the mapping between the (inferred) helicopter location and the prediction. Perseveration reduces the policy's complexity because it compresses the number of possible predictions stored in memory. This theory provides an appealing explanation of perseverative behavior in terms of resource rationality (Lieder and Griffiths, 2020). However, it would also require an extension to incorporate the increased environmental control of the responses. Taken together, our satisficing model shares theoretical ideas about perseveration with existing accounts but underlines the need for an extension of these models to cover the influence of external biases on learning. Importantly, our model highlights that the relationship between perseveration and environmental control of learning is a key factor explaining age-related differences in learning behavior of children and older adults compared to younger adults.

Perseveration is a common behavior in children and older adults that often leads to substantial performance limitations compared to younger adults. It has been observed in various experimental tasks in which performance relies on the flexible updating of task representations such as reversal learning (Crone et al., 2004a; Head et al., 2009; Ridderinkhof et al., 2002; Rutledge et al., 2009) or task switching (Blackwell and Munakata, 2014; Blackwell et al., 2014; Carroll et al., 2016; Cepeda and Munakata, 2007; Crone et al., 2004b; Munakata et al., 2012). Children's immature behavior in these tasks is often interpreted in terms of executive control and, in particular, working memory limitations. Our modeling approach offers an alternative interpretation of these limitations. These two age groups might more quickly be satisfied with perseverating on previous responses that led to sufficiently accurate outcomes during a stable task phase before a reversal of the contingencies or a task-switching cue.

Our finding of increased environmental control of learning in older adults is consistent with theoretical ideas that propose a shift from internal to environmental control with age (Craik and Bialystok, 2006; Lindenberger and Mayr, 2014). Lindenberger and Mayr suggest that across a range of psychological domains and experimental tasks, e.g., task switching, inhibitory working-memory, or attentional control, less differentiated internal representations in older adults are associated with an increased reliance on environmental information. Our model offers a formalization of this idea by assuming that external cues determine the value from which beliefs are updated. In effect, such cues bias the selected response towards the externally provided information. Because of a lower satisficing criterion, such biases are especially evident in older adults and children. So far, the environmental control framework has been primarily applied to the upper end of the lifespan. However, several findings from the developmental literature can be interpreted along the lines of this theory (Alarcón and Bonardi, 2020). For example, younger children tend to rely more on what has been referred to as reactive control (Chatham et al., 2009; Gonthier et al., 2019; Troller-Renfree et al., 2020). That is, they retrieve task-relevant information when needed (in response to an external cue) instead of maintaining the relevant information proactively (Munakata et al., 2012). In principle, our model reflects a similar distinction between a reactive mode characterized by a response to immediate task cues (being satisfied with cue-driven responses) and a proactive mode characterized by flexibly adjusting learning according to environmental statistics (normative belief updating).

Furthermore, it could be possible that a satisficing mechanism that is unknown to the experimenter and not considered as affecting behavior might be mistakenly attributed to random response noise. In particular, when environmental cues that externally determine the value from which beliefs are updated are subject to variability, this mechanism results in more response variability, a behavioral pattern that is often observed in children and older adults (Bonawitz et al., 2014; Li et al., 2001). Taken together, we think that the satisficing model may offer a parsimonious explanation for a wide array of findings on perseveration and environmental control in the developmental and aging literature.

Last but not least, we have not defined a trade-off between accuracy and potentially allocated cognitive resources that might determine satisficing criteria. Future studies should try to assess both cognitive costs 
and benefits to test whether individual differences in available resources are predictive of the degree of satisficing. Identifying a trade-off between them could lead to refined hypotheses about when people, given their limitations, should perseverate and rely more strongly on externally provided information. We leave the development of such a sophisticated resource-rational model to future work (e.g., Gershman (2020); Lieder and Griffiths (2020); Lieder et al. (2018)).

To conclude, in this study, we provide a unifying perspective on seemingly opposing internally and externally generated behavioral biases across the human lifespan. When updating beliefs from previous values, children and older adults frequently perseverate on previous predictions. In contrast, when beliefs are updated from externally manipulated values, learning in these age groups is more strongly controlled by the environment. We explain these effects with a satisficing model positing that belief updating is an active process through which the plausibility of a choice is sequentially evaluated until reaching a satisficing criterion. Our results suggest that children and older adults rely on a more liberal satisficing policy than younger adults and therefore show both stronger internal and external behavioral biases. The proposed satisficing model may offer a parsimonious explanation for a wide array of findings in the developmental and aging literature and supports a bounded rationality perspective on lifespan development.

\section{Methods}

\section{Participants}

For the first experiment, we recruited 33 children, 31 adolescents, 35 younger adults, and 37 older adults from the participant pools of the Chair of Lifespan Developmental Neuroscience at Technische Universität Dresden, with participants recruited from the Dresden community. Due to errors in the data acquisition, we excluded 2 adolescents and older adults, and 3 younger adults. The effective study sample consisted of 33 children ( 8 to 10 years, median age 9 years, 19 female), 29 adolescents (13 to 17 years, median age 15 years, 11 female), 32 younger adults (20 to 28 years, median age 24 years, 15 female), and 35 older adults (62 to 80 years, median age 71 years, 7 female). For the follow-up experiment, we recruited 34 children, 29 younger adults, and 38 older adults from the participant pool of the Max Planck Institute for Human Development Berlin. Due to errors in the data acquisition, we excluded 3 children and 4 younger and older adults. Here the effective study sample thus comprised 31 children ( 7 to 11 years, median age 9 years, 12 female), 25 younger adults (20 to 28 years, median age 24 years, 16 female), and 34 older adults (61 to 76 years, median age 67 years, 25 female). We measured the participants' processing speed and verbal knowledge using the Identical Picture and Spot-a-Word test (Lindenberger et al., 1993). In both experiments, we obtained written informed consent from the younger and older adults and from the parents of the children and, in the first experiment, the parents of the adolescents. The experimental protocol was approved by the ethics committee of the Max Planck Institute for Human Development Berlin.

\section{Experimental task}

We begin with an intuitive description of the task and the specific versions that we applied in the first and the follow-up experiment. After that, we provide a formal description of the task structure. The task was framed in an intuitive cover story, in which a helicopter dropped bags that had to be caught by participants (Fig. 2a). Because clouds covered the sky, participants could not observe the helicopter directly, but they were required to infer the helicopter position based on the previously dropped bags. Participants used a bucket that could be moved around via button presses to catch the bags (Prediction) using separate keys for fast movements (left: F, right: J) and slow movements (left: G, right: H). After participants had

placed their bucket, it could not be adjusted anymore. Subsequently, the hidden helicopter dropped a bag (Outcome). After that, a red line indicated the prediction error, defined as the distance between the 
actual and predicted location of the bag (Prediction error). Finally, participants updated their prediction of the next outcome (Update) and transitioned to the next trial.

The hidden helicopter represented the mean of a Gaussian distribution that generated the outcomes (Fig. 2b). The standard deviation of this distribution determined the amount of variability across outcomes, framed as wind in the cover story. As a consequence, bags mostly appeared left or right to the helicopter. Occasionally, the helicopter moved to a different location (changepoint). Therefore, the challenge in the task was the distinction of (a) systematic changes from (b) random influences. A prediction error could thus be due to (a) a changepoint, which should optimally result in a considerable adjustment of the bucket towards the most recent outcome or (b) random variability in the outcomes, which should lead to more subtle bucket adjustments. Only on a few trials, when the helicopter was visible, participants could distinguish changepoints and random variability to adjust their predictions to the mean of the outcome-generating distribution. Before performing the helicopter task, participants underwent a training session described in SI Task instructions.

FIRST EXPERIMENT In the first experiment, we used two conditions that differed in the amount of variability across outcomes. In the first condition, bags were only weakly corrupted by the wind. Consequently, changepoints could more easily be distinguished from random variability. In contrast, in the second, the wind was more forceful, and changepoints were harder to detect. These conditions were completed across four alternating task blocks, and the order of the conditions was counterbalanced between participants. Approximately half of the participants started with the low-variability condition, and the other half started with the high-variability condition. In the high-variability condition, participants achieve less accurate predictions compared to the low-variability condition. To approximately match the achieved performance between the conditions, we increased the bucket size in the second one. Moreover, bags randomly contained gold coins or stones. The number of collected gold coins was translated into monetary reward at the end of the task, but collected rocks were not rewarded. However, note that if gold or rocks were in the bag was only revealed after the bag was dropped. Consequently, to maximize the amount of reward, the best strategy was to try to catch every bag in the task. This reward structure of the task was explicitly communicated to the participants. Finally, for approximately half the participants, we inverted the reward value to control the potential salience effects of the color associated with bag content. Here, rewarding bags were labeled silver bags and neutral bags sandbags. Accordingly, participants were only rewarded for catching silver bags.

FOLLOW-UP EXPERIMENT In the follow-up experiment, our goal was to examine the influence of the learning environment and the reward associated with catching a bag on perseveration and estimation errors (Fig. 4a). We manipulated the learning environment by replacing the default position of the bucket. Before participants updated their predictions, we used a Gaussian distribution with a mean equal to the previously predicted bucket position to randomly determine the starting position of the bucket. To manipulate the amount of reward in the task, we indicated the bag content throughout the trial. In particular, this was displayed using a '+' symbol above the bucket, which was presented in gold (for gold content, high reward) or gray (for rocks, low reward). Finally, to focus on the two above-described manipulations, we kept the amount of variability in the outcomes constant. We used a variability level equal to the mean of the two conditions of the first experiment. This version of the task was divided into four task blocks (for all participants block 1 and 3: shifting-bucket environment; block 2, and 4: stable-bucket environment).

Formal DESCRIPTION A short note on the notation (Bruckner et al., 2019; Ostwald et al., 2018): We divide the formal documentation of our analyses into a task model, the description of the reduced Bayesian model, and the steps to evaluate and estimate the models based on behavioral data. We follow the conventions of applied probabilistic modeling, i.e., do not explicitly distinguish between probability dis- 
tributions, probability density functions, or probability mass functions. Moreover, we indicate parameters by superscripts.

- $T:=100$ denotes the number of trials for each of the four task blocks, indexed as $t=1,2, \ldots, T$,

- $X \in[0,300]$ denotes the set of outcomes $x$ in the task,

- $M \in[0,300]$ is the set of the mean parameters $\mu$ of the Gaussian distribution that generates the outcomes and that corresponds to the helicopter location in the cover story,

- $S \in \mathbb{N}_{>0}$ is the set of standard-deviation parameters of the Gaussian distribution that generates the outcomes, where in the first experiment, we have $\sigma=10$ (condition 1, less windy environment) and $\sigma=25$ (condition 2, more windy environment) and in the follow-up experiment $\sigma=17.5$,

- $C \in\{0,1\}$ is the set of changepoints in the outcome contingencies, where $c_{t}=0$ denotes that outcome contingencies are unchanged and $c_{t}=1$ indicates a changepoint in the outcome contingencies,

- $R \in\{0,1\}$ refers to the set of rewards in the task, where in the first experiment we used $r_{t}=0$ for no-reward trials and $r_{t}=1$ for high-reward trials; in the follow-up experiment, we used $r_{t}=0$ for low-reward and $r_{t}=1$ for high-reward trials,

- $V \in\{0,1\}$ refers to the set of helicopter-visibility trial types, where $v_{t}=0$ denotes that the helicopter was hidden and $v_{t}=1$ denotes that the helicopter was visible,

- $B \in[0,300]$ denotes the set of predictions $b$,

- $Z \in[0,300]$ denotes the set of default bucket locations $z$ in the follow-up experiment,

- $p^{h}\left(c_{t}\right):=\left\{\begin{array}{ll}0, & t \leq 3 \\ B\left(c_{t} ; h\right), & \text { otherwise }\end{array}\right.$ is the distribution that generates changepoints, i.e., changepoints do not occur for trials $t \leq 3$ and in all other cases according to the Bernoulli distribution, where $h:=0.125$ denotes the hazard rate that determines the frequency of changepoints,

- $p\left(\mu_{t} \mid c_{t}\right):=\left\{\begin{array}{ll}U\left(\mu_{t} ;[0,300]\right), & c_{t}=1 \\ \mu_{t-1}, & c_{t}=0\end{array}\right.$ is the changepoint-conditional distribution of $\mu_{t}$,

- $p^{\sigma^{2}}\left(x_{t} \mid \mu_{t}\right):=N\left(x_{t} ; \mu_{t}, \sigma^{2}\right)$ is the outcome-generating Gaussian distribution, where $x_{t}<0$ and $x_{t}>300$ were adjusted to $x_{t}=0$ and $x_{t}=300$ respectively, in order to adjust the outcomes to the edges of the task screen,

- $p\left(r_{t}\right):=B\left(r_{t} ; 0.5\right)$ is a Bernoulli distribution that generates the reward type (high versus low reward),

- $p\left(v_{t}\right):=B\left(c_{t} ; 0.1\right)$ is a Bernoulli distribution that determines the helicopter visibility, and finally,

- $p\left(z_{t} \mid b_{t-1}\right):=N\left(z_{t} ; b_{t-1}, 20^{2}\right)$ denotes the Gaussian distribution that determined the default bucket location in the shifting-bucket environment of the follow-up experiment, which was similarly constrained between 0 and 300 as the outcomes. 


\section{Data preprocessing}

Additional variables of interest for the analyses were participants' estimation errors, prediction errors, prediction updates, and perseveration trials. In the follow-up experiment, we were additionally interested in the shift in the default bucket position.

- $D \in[-300,300]$ refers to the set of prediction errors, defined as $\delta_{t}:=x_{t}-b_{t}$,

- $E \in[0,300]$ is the set of estimation errors, defined as $e_{t}:=\left|\mu_{t}-b_{t}\right|$, i.e., the absolute distance between the helicopter location and the prediction,

- $A \in[-300,300]$ refers to the set of trial-by-trial prediction updates, which are defined as, $a_{t}:=$ $b_{t+1}-b_{t}$

- PERS $\in\{0,1\}$ refers to the set of perseveration trials, defined as $\operatorname{per}_{t}:= \begin{cases}1, a_{t}=0 & \text { i.e., } \\ 0, \text { otherwise, } & \end{cases}$ trials in which predictions were not updated were categorized as perseveration trials,

- MOTORPERS $\in\{0,1\}$ refers to the set of trials, in which participants directly locked in their prediction at the default bucket location. Motor perseveration was defined as motor_pers $s_{t}:=$ $\left\{\begin{array}{l}1, b_{t}=z_{t} \\ 0, \text { otherwise, }\end{array}\right.$ and only of interest in the follow-up experiment, and finally,

- $Y \in[-300,300]$ refers to the shift in the default bucket location, defined as $y_{t}:=z_{t+1}-b_{t}$.

\section{Average learning-rate analysis}

For each participant, we computed the average learning rate using the regression model

$$
a_{t}=\beta_{0}+\beta_{1} \delta_{t}
$$

where $a_{t}$ refers to the prediction update at trial $t, \beta_{0}$ to the intercept, and $\beta_{1}$ to the learning rate that determined the average influence of the prediction error $\delta_{t}$ on the update $a_{t}$.

In the follow-up experiment, we additionally took into consideration the shift in the default bucket location $y_{t}$

$$
a_{t}=\beta_{0}+\beta_{1} \delta_{t}+\beta_{2} y_{t} .
$$

To estimate the free parameters, we used ordinary least squares from the statsmodels OLS class in Python.

\section{Robust regression}

In the follow-up experiment, we computed the association between perseveration in the stable-bucket environment and the belief-updating bias in the shifting-bucket environment using the robust linear regression model

$$
d_{i}=\beta_{0}+\beta_{1} \hat{p}(\text { pers })_{i}
$$

where $d_{i}$ denotes the estimated belief-updating bias of participant $i, \beta_{0}$ denotes the intercept of the regression model, and $\beta_{1}$ the regression weight of $\hat{p}(\text { pers })_{i}$, i.e., the estimated perseveration probability of participant $i$. In Fig. $4 \mathrm{~h}$, the points show $d_{i}$ as a function of $\hat{p}(\text { pers })_{i}$ and the line shows the predicted values according to the robust regression model (see also SI Robust regression with age group). We estimated the model using robust regression from statsmodels in Python. 


\section{Reduced Bayesian model}

The experimental task can be solved using a Bayesian changepoint-detection algorithm that predicts future outcomes based on all previous outcomes (Adams and MacKay, 2007; Wilson et al., 2010). This solution requires considering all possible combinations of changepoints (see SI Optimal and approximate inference). However, given the unrealistic assumption that humans similarly rely on the memory of all previous trials during learning, Nassar and colleagues proposed a reduced learning model that approximates this algorithm (Nassar et al., 2010, 2012; Wilson et al., 2010).

In the reduced Bayesian model, the learning-rate regulation depends on relative uncertainty, changepoint probability, and hazard rate. Relative uncertainty indicates the uncertainty of the model's belief about the helicopter location based on the history of outcomes relative to the variability in the environment. When the model has only observed a few outcomes, relative uncertainty tends to be higher and subsequently declines with each new observation (Fig. 2b). After a changepoint, the previously learned helicopter estimate is not predictive of future outcomes anymore. Therefore the best strategy is to re-start the estimation of the helicopter position. However, because the model can not directly observe changepoints, it keeps track of changepoint probability, which indicates the probability of a changepoint based on the most recently observed outcome (Fig. 2b). Changepoint probability itself depends on the hazard-rate parameter, which reflects the model's assumption about the rate of change in the environment. That is, with a subjectively higher hazard-rate parameter, the model assumes more changepoints throughout the task. The learning rate itself is a weighted combination of relative uncertainty and changepoint probability (Fig. 2b). When changepoint probability is high, more recent outcomes have a greater impact on learning, whereas more distant outcomes are forgotten. When changepoint probability is low, learning is mostly driven by relative uncertainty, and the model more strongly considers multiple previous outcomes.

In contrast to the task model, $\mu_{t}$ (i.e., helicopter location) is unknown to the model and has to be learned throughout the task. Here

- $\mu_{0}:=150$ is the model's initial estimate of the $\mu$ parameter,

- $\sigma_{0}^{2}:=100$ is the model's initial estimation uncertainty over the $\mu$ parameter,

- $u \in \mathbb{R}_{\geq 0}$ is the uncertainty-underestimation parameter, indicating the extent to which estimation uncertainty is underestimated ( $u=0$ if the model does not suffer from uncertainty underestimation),

- $h \in[0,1]$ is the subjective hazard-rate parameter that models participants' assumptions about the overall probability of a changepoint,

- $s \in[0,1]$ is the surprise-sensitivity parameter that indicates the degree to which changepoint probability influences the learning rate; hence higher values indicate a stronger influence of changepoint probability on learning ( $s=1$ if the model does not suffer from reduced surprise sensitivity),

- $\mu_{H}$ is the true location of the helicopter that is only revealed during catch trials,

- $\sigma_{H} \in \mathbb{N}_{>0}$ is a free parameter that models the standard deviation of the Gaussian distribution of the perceptual information provided by the visible helicopter $\left(\sigma_{H}=0\right.$ if the model perfectly considers catch trials),

- $q \in[-1,1]$ is a free parameter (in the first experiment) that determines the degree to which the reward type of the previous trial modulates the learning rate $(q=0$ if the model is not influenced by reward), finally, 
- $d \in \mathbb{R}$ is a free parameter (in the follow-up experiment) that models the influence of the shift in the default bucket location $y_{t}$ on the belief update $(d=0$ if the shifted bucket does not bias the model).

To infer the $\mu_{t+1}$ parameter, the reduced Bayesian model combines the latest outcome $x_{t}$ and the previous prediction $\mu_{t}$. This combination is achieved using an error-driven sequential updating rule

$$
\mu_{t+1}:=\mu_{t}+\hat{a}_{t}
$$

where

$$
\hat{a}_{t}:=\alpha_{t} \delta_{t}
$$

and where

$$
\delta_{t}:=x_{t}-\mu_{t}
$$

refers to the prediction error that expresses the difference between the last outcome $x_{t}$ and the last prediction $\mu_{t}$. The weight of this combination is determined by the learning rate $\alpha_{t}$. The reduced Bayesian model uses a dynamical learning rate that depends on the two factors changepoint probability $\omega_{t}$ and relative uncertainty $\tau_{t}$ :

$$
\alpha_{t}:=\omega_{t}+\tau_{t}-\tau_{t} \omega_{t}
$$

Changepoint probability $\omega_{t}$ is computed as a function of the current prediction error $\delta_{t}$, the assumed hazard rate $h$, the total uncertainty about the next outcome, and the degree of surprise sensitivity $s$, i.e.,

$$
\omega_{t}:=\frac{\left(U\left(\delta_{t} ;[0,300]\right)\right)^{s} h}{\left(N\left(\delta_{t} ; 0, \sigma_{t}^{2}+\sigma^{2}\right)\right)^{s}(1-h)+\left(U\left(\delta_{t} ;[0,300]\right)\right)^{s} h} .
$$

Next, to determine relative uncertainty $\tau_{t+1}$ for the next trial, the model first computes the estimation uncertainty about the helicopter position, defined as

$$
\sigma_{t+1}^{2}:=\frac{\omega_{t} \sigma^{2}+\left(1-\omega_{t}\right) \tau_{t} \sigma^{2}+\omega_{t}\left(1-\omega_{t}\right)\left(\delta_{t}\left(1-\tau_{t}\right)\right)^{2}}{\exp (u)}
$$

where the $u$ parameter determines the degree of uncertainty underestimation.

Subsequently, the model computes the fraction of estimation uncertainty relative to its total uncertainty about the next outcome, which is the sum of estimation uncertainty and the variability in the environment $\sigma^{2}$

$$
\tau_{t+1}:=\frac{\sigma_{t+1}^{2}}{\sigma_{t+1}^{2}+\sigma^{2}} .
$$

When the helicopter was occasionally presented, we assumed that participants would partially consider the additional information to adjust their helicopter estimate and the associated estimation uncertainty (see SI Catch-trial derivations). During catch trials, the inferred helicopter location was a combination of the previously learned helicopter position and the true helicopter location

$$
\mu_{t+1}=\left(1-w_{t}\right) \mu_{t+1}+w_{t} \mu_{H},
$$


where

$$
w_{t}:=\frac{\sigma_{t}^{2}}{\sigma_{t}^{2}+\sigma_{H}^{2}},
$$

and where $\sigma_{H}^{2}$ models to which degree the reduced Bayesian model trusts the helicopter information (i.e., higher $\sigma_{H}^{2}$ leads to a lower consideration of catch trials). Relative uncertainty was subsequently adjusted to incorporate the additional information provided by the visible helicopter

$$
\tau_{t}=\frac{C}{C+\sigma^{2}}
$$

where

$$
C:=\frac{1}{\frac{1}{\sigma_{t}^{2}}+\frac{1}{\sigma_{H}^{2}}} .
$$

Moreover, to model a reward-driven learning-rate bias in trials in which the previous reward was high $\left(r_{t-1}=1\right.$, in the first experiment) (McGuire et al., 2014), we added the value of the freely estimated reward-bias parameter to the learning rate $\left(\alpha_{t}+q\right)$ and for all $\alpha_{t}>1$ and $\alpha_{t}<0$ adjusted the learning rate to $\alpha_{t}=1$ and $\alpha_{t}=0$ respectively.

Finally, in the follow-up experiment we modeled the influence of the distorted default bucket location $y_{t}$ on the belief adjustment $\hat{a}_{t}$ with the additional free parameter $d$ :

$$
\hat{a}_{t}=\hat{a}_{t}+d y_{t}
$$

\section{Perseveration model}

To model perseveration, we used the Dirac-delta function

$$
\delta\left(a_{t}\right)= \begin{cases}1, & a_{t}=0 \\ 0, & \text { otherwise }\end{cases}
$$

according to which perseveration probability is 0 everywhere except at 0 , where it is 1 . As described in the following section, we combined this perseveration model with the reduced Bayesian model, and we estimated the contribution of each model to updating behavior for each participant.

\section{Evaluation and estimation}

We evaluated and estimated the mixture model using participants' prediction errors. That is, instead of computing a prediction error as the difference between the actual outcome $x_{t}$ and the learned $\mu_{t}$ parameter of the reduced Bayesian model (see Eq. (6)), we used the actual prediction errors of our participants as described in Data preprocessing. Using the participants' prediction errors is advantageous over modelbased prediction errors because participant and model predictions might occasionally deviate from each other. Next to the free parameters described above, we estimated the following parameters:

- $o_{0} \in \mathbb{N}_{>0}$ is the motor-noise parameter, modeling noise in the placement of the bucket,

- $o_{1} \in \mathbb{N}_{>0}$ is the learning-rate noise parameter that accounts for increasing noise as a function of the required update magnitude,

- $b_{0} \in \mathbb{N}$ is the intercept of the logistic function that determined the mixture weight between the learning and perseveration component, 
- $b_{1} \in \mathbb{N}$ is the slope of the same logistic function.

To evaluate the probability of the current update, we used a Gaussian distribution

$$
p\left(a_{t} \mid x_{1: T}\right):=N\left(a_{t} ; \hat{a}_{t}, \varepsilon_{t}^{2}\right)
$$

where

$$
\varepsilon_{t}:=o_{0}+o_{1}\left|\hat{a}_{t}\right|
$$

Finally, the mixture between the probability of the learning component and the probability of the perseveration component was determined by

$$
p^{\lambda_{t}, \delta}\left(a_{t} \mid x_{1: T}\right):=\lambda_{t} \delta\left(a_{t}\right)+\left(1-\lambda_{t}\right) p\left(a_{t} \mid x_{1: T}\right),
$$

where

$$
\lambda_{t}:=\frac{1}{1+\exp \left(-b_{1}\left(\left|\hat{a}_{t}\right|-b_{0}\right)\right)}
$$

We assumed that trials with an update equal to zero could be due to the learning component or the perseveration component (where the higher the absolute predicted update of the reduced Bayesian model, the lower the probability that the trial was generated from the perseveration component). In contrast, for trials where participants did not perseverate, we assumed that data were exclusively generated from the learning component.

We estimated the free parameters using the constrained minimization algorithm L-BFGS-B from the Python SciPy optimize framework. We repeated the estimation 25 times using randomly determined starting points and selected the parameters of the iteration that achieved the best fit to the data, i.e., the lowest negative log-likelihood. To ensure reproducible results, we used the same, fixed value of the random seed for all analyses. Finally, consistent with our previous study (Nassar et al., 2016), we used a weakly informative Gaussian prior with mean 0 and standard deviation 5 to regularize the estimation of the uncertainty-underestimation parameter.

\section{Satisficing model}

In the satisficing model, we first computed the spatial movement

$$
m_{t}:=-y_{t}+a_{t}^{*},
$$

that is, the optimal belief update according to the reduced Bayesian model $a_{t}^{*}$ under consideration of the shift in the default bucket location $y_{t}$. The movement $m_{t}$ thus expresses the distance of the required bucket adjustment to compensate for the shift in the default bucket location.

To model the influence of the default belief, we exploited the Gaussian distribution representing the model's belief over the helicopter location, where the mean corresponds to the most likely location of the helicopter, and the variance represents the model's belief (or estimation) uncertainty, which we fixed to $\sigma_{t}^{2}=20^{2}$ in the satisficing simulations. The normative reduced Bayesian model without default belief would report an update towards the mean $a_{t}^{*}$ of this distribution (Fig. 5a). However, we assumed that the satisficing model reports the default belief when the optimal belief update is close to the default, which was determined by the criterion $j$ (Fig. 5b-d). To translate the criterion $j$ into perseveration or environmentally biased updating, we used the Gaussian percent-point function (Q) (also known as quantile function; see SI Percent-point function). 
We evaluated if the required bucket movement $m_{t}$ was below the satisficing criterion, leading to perseveration or above, leading to an update. We computed the current criterion value according to

$$
w_{t}:=\left\{\begin{array}{l}
Q(j), m_{t} \geq 0 \\
Q(1-j), m_{t}<0 .
\end{array}\right.
$$

This case distinction indicates that for a positive $m_{t}$, reflecting a rightward movement on the screen, we considered the left part of the distribution and vice versa for a negative $m_{t}$. The reported belief update was then determined according to

$$
a_{t}= \begin{cases}y_{t}, & \operatorname{sgn}\left(w_{t}\right) \neq \operatorname{sgn}\left(m_{t}\right) \\ N\left(y_{t}+w_{t}, \epsilon^{2}\right), & \text { otherwise, }\end{cases}
$$

i.e., when the criterion value $w_{t}$ and the required movement $m_{t}$ had an unequal sign (i.e., the default belief is above the criterion), the model's belief update was equal to the default bucket location. In the stablebucket environment, this led to perseveration, and in the shifting-bucket environment to a prediction equal to the bucket's externally determined default position. When signs were equal (i.e., the default belief is below the criterion), we modeled a belief update that led to a prediction at the computed criterion value under the addition of Gaussian noise $\epsilon=5$. See SI Satisficing model for more details and an illustration of the model.

\section{Software and data repository}

Pseudonymized experimental data in brain imaging data structure (BIDS) format (Gorgolewski et al., 2016) and the Python analysis code are available at

https://github.com/rasmusbruckner/adaptivelearning_analysis.

The task was programmed in Matlab (The Mathworks Inc., USA) using the Psychtoolbox2 (Brainard, 1997; Pelli, 1997; Kleiner et al., 2007), MGL (http://gru.stanford.edu/doku.php?id=mgl:overview) and SnowDots (http://code.google.com/p/snow-dots) extensions. Data analysis and computational modeling was performed in Python 3.6 (Python Software Foundation; https://www.python.org/). We used the NumPy (Oliphant, 2006; Van Der Walt et al., 2011), SciPy (Virtanen et al., 2020), pandas (McKinney, 2010), statsmodels (Seabold and Perktold, 2010), matplotlib (Hunter, 2007), seaborn (https: //doi.org/10.5281/zenodo.12710), PIL (https://pillow.readthedocs.io/en/stable/index.html), and tqdm (https://doi.org/10.5281/zenodo.1239851) libraries. Protected exceedance probabilities were computed in SPM12 (Penny et al., 2011) using Matlab.

\section{Acknowledgements}

We thank Ulman Lindenberger for helpful comments and providing the testing infrastructure at the Max Planck Institute for Human Development Berlin. We also thank Michael J. Frank, Dirk Ostwald, and Jan Bruckner for helpful comments, Ben Heasly for programming the task, and Lennart Wittkuhn, Sabrina Wideburg, Lola Herrmann, and Lina Nguyen for their help during data acquisition. R.B. was supported by the International Max Planck Research School LIFE, Berlin, Germany, and by a PROMOS stipend from the German Academic Exchange Service (DAAD). M.R.N. was supported by NIH grant F32-MH10200901A1 and a travel grant provided by the Burroughs Welcome Fund. S.-C.L. and B.E. were supported by grants from the German Federal Ministry of Education and Research (BMBF; FKZ 01GQ0913, FKZ 01GQ1313). B.E. received support from the German Research Foundation (DFG; SFB 940/2 B7), as well as the Natural Sciences and Engineering Research Council of Canada (NSERC; N01882). Furthermore, this research was undertaken, in part, thanks to funding from the Canada Research Chairs program. 


\section{Author contributions}

R.B., M.R.N., S.-C.L., and B.E. designed the study, R.B. and B.E. collected the data, R.B. and M.R.N. analyzed the data. R.B. wrote the manuscript. R.B., M.R.N., S.-C.L., and B.E. reviewed and edited the manuscript. B.E. and S.-C.L. funded the study.

\section{Conflict of interest statement}

The authors declare no conflict of interest. 


\section{References}

Adams, R. P. and MacKay, D. J. C. (2007). Bayesian online changepoint detection. arXiv.

Alarcón, D. E. and Bonardi, C. (2020). Under the influence of the environment: Children's responding invigorated and biased by predictive cues. Journal of Experimental Child Psychology, 191:104741.

Behrens, T. E., Woolrich, M. W., Walton, M. E., and Rushworth, M. F. (2007). Learning the value of information in an uncertain world. Nature Neuroscience, 10(9):1214-1221.

Blackwell, K. A., Chatham, C. H., Wiseheart, M., and Munakata, Y. (2014). A developmental window into trade-offs in executive function: The case of task switching versus response inhibition in 6-year-olds. Neuropsychologia, 62:356-364.

Blackwell, K. A. and Munakata, Y. (2014). Costs and benefits linked to developments in cognitive control. Developmental Science, 17(2):203-211.

Bolenz, F., Reiter, A. M. F., and Eppinger, B. (2017). Developmental changes in learning: Computational mechanisms and social influences. Frontiers in Psychology, 8, 2048.

Bonawitz, E., Denison, S., Griffiths, T. L., and Gopnik, A. (2014). Probabilistic models, learning algorithms, and response variability: Sampling in cognitive development. Trends in Cognitive Sciences, 18(10):497-500.

Brainard, D. H. (1997). The psychophysics toolbox. Spatial Vision, 10(4):433-436.

Bruckner, R., Heekeren, H. R., and Ostwald, D. (2019). Perceptual uncertainty modulates human rewardbased learning. Conference on Cognitive Computational Neuroscience.

Carroll, D. J., Blakey, E., and FitzGibbon, L. (2016). Cognitive flexibility in young children: Beyond perseveration. Child Development Perspectives, 10(4):211-215.

Cepeda, N. J. and Munakata, Y. (2007). Why do children perseverate when they seem to know better: Graded working memory, or directed inhibition? Psychonomic Bulletin ES Review, 14(6):1058-1065.

Chatham, C. H., Frank, M. J., and Munakata, Y. (2009). Pupillometric and behavioral markers of a developmental shift in the temporal dynamics of cognitive control. Proceedings of the National Academy of Sciences, 106(14):5529-5533.

Chowdhury, R., Guitart-Masip, M., Lambert, C., Dayan, P., Huys, Q. J. M., Düzel, E., and Dolan, R. J. (2013). Dopamine restores reward prediction errors in old age. Nature Neuroscience, 16(5):648-653.

Collins, A. G. E. and Cockburn, J. (2020). Beyond dichotomies in reinforcement learning. Nature Reviews Neuroscience, 21(10):576--586.

Craik, F. I. M. and Bialystok, E. (2006). Cognition through the lifespan: Mechanisms of change. Trends in Cognitive Sciences, 10(3):131-138.

Crone, E. A., Jennings, J. R., and Van der Molen, M. W. (2004a). Developmental change in feedback processing as reflected by phasic heart rate changes. Developmental Psychology, 40(6):1228-1238.

Crone, E. A., Ridderinkhof, R. K., Worm, M., Somsen, R. J. M., and van der Molen, M. W. (2004b). Switching between spatial stimulus-response mappings: A developmental study of cognitive flexibility. Developmental Science, 7(4):443-455. 
Daw, N. D. (2014). Advanced reinforcement learning. In Neuroeconomics, pages 299-320. Academic Press.

Dayan, P. and Daw, N. D. (2008). Decision theory, reinforcement learning, and the brain. Cognitive, Affective, \& Behavioral Neuroscience, 8(4):429-453.

De Boer, L., Axelsson, J., Riklund, K., Nyberg, L., Dayan, P., Bäckman, L., and Guitart-Masip, M. (2017). Attenuation of dopamine-modulated prefrontal value signals underlies probabilistic reward learning deficits in old age. eLife, 6:e26424.

Eppinger, B., Kray, J., Mock, B., and Mecklinger, A. (2008). Better or worse than expected? Aging, learning, and the ERN. Neuropsychologia, 46(2):521-539.

Eppinger, B., Mock, B., and Kray, J. (2009). Developmental differences in learning and error processing: Evidence from ERPs. Psychophysiology, 46(5):1043-1053.

Eppinger, B., Schuck, N. W., Nystrom, L. E., and Cohen, J. D. (2013). Reduced striatal responses to reward prediction errors in older compared with younger adults. Journal of Neuroscience, 33(24):99059912.

Gershman, S. J. (2020). Origin of perseveration in the trade-off between reward and complexity. Cognition, page 104394.

Gigerenzer, G. and Gaissmaier, W. (2011). Heuristic decision making. Annual Review of Psychology, 62:451-482.

Gonthier, C., Zira, M., Colé, P., and Blaye, A. (2019). Evidencing the developmental shift from reactive to proactive control in early childhood and its relationship to working memory. Journal of Experimental Child Psychology, 177:1-16.

Gorgolewski, K. J., Auer, T., Calhoun, V. D., Craddock, R. C., Das, S., Duff, E. P., ..., and Poldrack, R. A. (2016). The brain imaging data structure, a format for organizing and describing outputs of neuroimaging experiments. Scientific Data, 3(1):1-9.

Hämmerer, D., Li, S.-C., Müller, V., and Lindenberger, U. (2011). Life span differences in electrophysiological correlates of monitoring gains and losses during probabilistic reinforcement learning. Journal of Cognitive Neuroscience, 23(3):579-592.

Hämmerer, D., Schwartenbeck, P., Gallagher, M., FitzGerald, T. H. B., Düzel, E., and Dolan, R. J. (2019). Older adults fail to form stable task representations during model-based reversal inference. Neurobiology of Aging, 74:90-100.

Head, D., Kennedy, K. M., Rodrigue, K. M., and Raz, N. (2009). Age differences in perseveration: Cognitive and neuroanatomical mediators of performance on the Wisconsin Card Sorting Test. Neuropsychologia, 47(4):1200-1203.

Hunter, J. D. (2007). Matplotlib: A 2d graphics environment. Computing in Science $E$ Engineering, $9(3): 90-95$.

Kleiner, M., Brainard, D. H., and Pelli, D. G. (2007). What's new in Psychtoolbox 3? In Perception 36 ECVP Abstract Supplement. 
Li, S.-C., Lindenberger, U., Hommel, B., Aschersleben, G., Prinz, W., and Baltes, P. B. (2004). Transformations in the couplings among intellectual abilities and constituent cognitive processes across the life span. Psychological Science, 15(3):155-163.

Li, S.-C., Lindenberger, U., and Sikström, S. (2001). Aging cognition: From neuromodulation to representation. Trends in Cognitive Sciences, 5(11):479-486.

Lieder, F. and Griffiths, T. L. (2020). Resource-rational analysis: Understanding human cognition as the optimal use of limited computational resources. Behavioral and Brain Sciences, e1:1-60.

Lieder, F., Griffiths, T. L., Huys, Q. J. M., and Goodman, N. D. (2018). The anchoring bias reflects rational use of cognitive resources. Psychonomic Bulletin $\&$ Review, 25(1):322-349.

Lindenberger, U. and Mayr, U. (2014). Cognitive aging: Is there a dark side to environmental support? Trends in Cognitive Sciences, 18(1):7-15.

Lindenberger, U., Mayr, U., and Kliegl, R. (1993). Speed and intelligence in old age. Psychology and Aging, 8(2):207-220.

McGuire, J. T., Nassar, M. R., Gold, J. I., and Kable, J. W. (2014). Functionally dissociable influences on learning rate in a dynamic environment. Neuron, 84(4):870-881.

McKinney, W. (2010). Data Structures for statistical computing in Python. In Proceedings of the 9th Python in Science Conference, volume 445, pages 51-56.

Meyniel, F. and Dehaene, S. (2017). Brain networks for confidence weighting and hierarchical inference during probabilistic learning. Proceedings of the National Academy of Sciences, 114(19):E3859-E386.

Miller, K. J., Shenhav, A., and Ludvig, E. A. (2019). Habits without values. Psychological Review, 126(2):292.

Munakata, Y., Snyder, H. R., and Chatham, C. H. (2012). Developing cognitive control: Three key transitions. Current Directions in Psychological Science, 21(2):71-77.

Nassar, M. R., Bruckner, R., Gold, J. I., Li, S.-C., Heekeren, H. R., and Eppinger, B. (2016). Age differences in learning emerge from an insufficient representation of uncertainty in older adults. Nature Communications, 7:11609.

Nassar, M. R. and Frank, M. J. (2016). Taming the beast: Extracting generalizable knowledge from computational models of cognition. Current Opinion in Behavioral Sciences, 11:49-54.

Nassar, M. R., Rumsey, K. M., Wilson, R. C., Parikh, K., Heasly, B., and Gold, J. I. (2012). Rational regulation of learning dynamics by pupil-linked arousal systems. Nature Neuroscience, 15(7):1040.

Nassar, M. R., Wilson, R. C., Heasly, B., and Gold, J. I. (2010). An approximately Bayesian delta-rule model explains the dynamics of belief updating in a changing environment. Journal of Neuroscience, 30(37):12366-12378.

Oliphant, T. E. (2006). A Guide to NumPy. USA: Trelgol Publishing.

Ostwald, D., Bruckner, R., and Heekeren, H. R. (2018). Computational mechanisms of human state-actionreward contingency learning under perceptual uncertainty. Conference on Cognitive Computational Neuroscience. 
O’Reilly, J. X., Schüffelgen, U., Cuell, S. F., Behrens, T. E. J., Mars, R. B., and Rushworth, M. F. S. (2013). Dissociable effects of surprise and model update in parietal and anterior cingulate cortex. Proceedings of the National Academy of Sciences, 110(38):E3660-E3669.

Palminteri, S., Wyart, V., and Koechlin, E. (2017). The importance of falsification in computational cognitive modeling. Trends in Cognitive Sciences, 21(6):425-433.

Payzan-LeNestour, E., Dunne, S., Bossaerts, P., and O'Doherty, J. P. (2013). The neural representation of unexpected uncertainty during value-based decision making. Neuron, 79(1):191-201.

Pelli, D. G. (1997). The VideoToolbox software for visual psychophysics: Transforming numbers into movies. Spatial Vision, 10(4):437-442.

Penny, W. D., Friston, K. J., Ashburner, J. T., Kiebel, S. J., and Nichols, T. E. (2011). Statistical Parametric Mapping: The Analysis of Functional Brain Images. Elsevier.

Piray, P. and Daw, N. D. (2020). Linear reinforcement learning: Flexible reuse of computation in planning, grid fields, and cognitive control. bioRxiv.

Ridderinkhof, K. R., Span, M. M., and Van Der Molen, M. W. (2002). Perseverative behavior and adaptive control in older adults: Performance monitoring, rule induction, and set shifting. Brain and cognition, 49(3):382-401.

Rigoux, L., Stephan, K. E., Friston, K. J., and Daunizeau, J. (2014). Bayesian model selection for group studies - Revisited. NeuroImage, 84:971-985.

Rutledge, R. B., Lazzaro, S. C., Lau, B., Myers, C. E., Gluck, M. A., and Glimcher, P. W. (2009). Dopaminergic drugs modulate learning rates and perseveration in Parkinson's patients in a dynamic foraging task. Journal of Neuroscience, 29(48):15104-15114.

Samanez-Larkin, G. R., Kuhnen, C. M., Yoo, D. J., and Knutson, B. (2010). Variability in nucleus accumbens activity mediates age-related suboptimal financial risk taking. Journal of Neuroscience, $30(4): 1426-1434$.

Samanez-Larkin, G. R., Levens, S. M., Perry, L. M., Dougherty, R. F., and Knutson, B. (2012). Frontostriatal white matter integrity mediates adult age differences in probabilistic reward learning. Journal of Neuroscience, 32(15):5333-5337.

Samanez-Larkin, G. R., Worthy, D. A., Mata, R., McClure, S. M., and Knutson, B. (2014). Adult age differences in frontostriatal representation of prediction error but not reward outcome. Cognitive, Affective $\&$ Behavioral Neuroscience, 14(2):672-682.

Seabold, S. and Perktold, J. (2010). statsmodels: Econometric and statistical modeling with python. In 9th Python in Science Conference.

Simon, H. A. (1955). A behavioral model of rational choice. The Quarterly Journal of Economics, 69(1):99-118.

Simon, H. A. (1956). Rational choice and the structure of the environment. Psychological Review, 63(2):129-138.

Stephan, K. E., Penny, W. D., Daunizeau, J., Moran, R. J., and Friston, K. J. (2009). Bayesian model selection for group studies. NeuroImage, 46(4):1004-1017. 
Troller-Renfree, S. V., Buzzell, G. A., and Fox, N. A. (2020). Changes in working memory influence the transition from reactive to proactive cognitive control during childhood. Developmental Science, page e12959.

Vaghi, M. M., Luyckx, F., Sule, A., Fineberg, N. A., Robbins, T. W., and De Martino, B. (2017). Compulsivity reveals a novel dissociation between action and confidence. Neuron, 96(2):348-354.

Van den Bos, W., Cohen, M. X., Kahnt, T., and Crone, E. A. (2012). Striatum-medial prefrontal cortex connectivity predicts developmental changes in reinforcement learning. Cerebral Cortex, 22(6):12471255 .

Van der Schaaf, M. E., Warmerdam, E., Crone, E. A., and Cools, R. (2011). Distinct linear and non-linear trajectories of reward and punishment reversal learning during development: Relevance for dopamine's role in adolescent decision making. Developmental Cognitive Neuroscience, 1(4):578-590.

Van Der Walt, S., Colbert, S. C., and Varoquaux, G. (2011). The NumPy array: A structure for efficient numerical computation. Computing in Science \& Engineering, 13(2):22-30.

Virtanen, P., Gommers, R., Oliphant, T. E., Haberland, M., Reddy, T., Cournapeau, D., Burovski, E., Peterson, P., Weckesser, W., Bright, J., van der Walt, S. J., Brett, M., Wilson, J., Jarrod Millman, K., Mayorov, N., Nelson, A. R. J., Jones, E., Kern, R., Larson, E., Carey, C., Polat, İ., Feng, Y., Moore, E. W., Vand erPlas, J., Laxalde, D., Perktold, J., Cimrman, R., Henriksen, I., Quintero, E. A., Harris, C. R., Archibald, A. M., Ribeiro, A. H., Pedregosa, F., van Mulbregt, P., and Contributors, S. . . (2020). SciPy 1.0: Fundamental algorithms for scientific computing in Python. Nature Methods, 17:261-272.

Wilson, R. C., Nassar, M. R., and Gold, J. I. (2010). Bayesian online learning of the hazard rate in change-point problems. Neural Computation, 22(9):2452-2476.

Wood, W. and Rünger, D. (2016). Psychology of habit. Annual Review of Psychology, 67:289-314. 


\section{Supplementary information}

\section{Task instructions}

Before entering the experimental task, participants were instructed verbally and using written material on the computer screen. We built up the helicopter cover story step-by-step, with practice blocks in between. Every practice phase consisted of a low- and high-variability block. In the first practice phase, the helicopter was always visible. To ensure that participants understood that the helicopter is the best predictor of the outcomes, we used a response criterion that required participants to put their bucket ten times exactly underneath the visible helicopter. These blocks either stopped after the criterion was reached or after a maximum of 80 trials. In the second practice phase, clouds covered the helicopter, and participants had to infer its location based on the outcomes. Occasionally, during catch trials, the helicopter became visible, and participants could use this information for learning. Both of these blocks consisted of 50 trials and were the same as in the real experiment. After the practice session, we emphasized again that participants would earn money for their collected points. 


\section{Computational modeling first experiment}

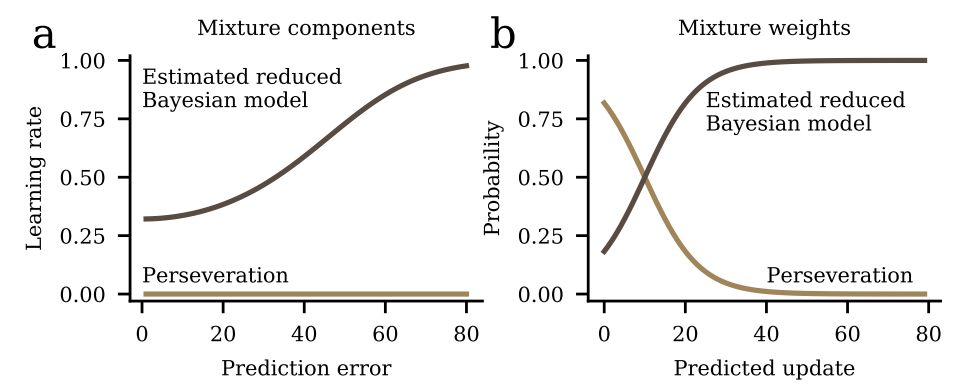

Figure SM 1. Mixture model. To account for age-related differences in perseveration within our model, the model contained a perseveration component that allowed for a better explanation of participant data than a control model without perseveration. a) In our task, perseveration can computationally be described as a learning rate $\alpha_{t}=0$, which leads the model to ignore the prediction error and consequently, to an exact repetition of the previous prediction. b) We modeled the contribution of this perseveration component (perseveration probability) as a function of the predicted update of the reduced Bayesian model. In effect, the model predicted a higher perseveration probability during smaller prediction errors. 


\section{Mixture model}

To better understand the computational underpinnings of differences in learning we analyzed trial-bytrial dynamics of learning-rate adjustments in the different age groups using the mixture model. The uncertainty-underestimation parameter reflects the extent to which participants underestimate uncertainty during learning. Higher uncertainty-underestimation parameters lead to lower learning rates in the range of small prediction errors that are most likely due to variability in environment (Fig. SM 2a). The mostly positive parameter estimates reflecting uncertainty underestimation shown in Fig. SM 2 d suggest that all age groups tended to underestimate uncertainty, because a full consideration of uncertainty is indicated with an uncertainty-underestimation parameter equal to 0. We next used an ANOVA to compare uncertainty underestimation across the four age groups, which did not show significant parameter differences between children (median $=3.878$, IQR 2.193 to 5.191), adolescents (median $=2.791$, IQR 1.633 to 4.274), younger adults (median $=2.43$, IQR 1.764 to 4.263 ), and older adults (median $=3.574$, IQR 2.458 to 4.444) $(H=6.308, p=0.098)$. However, because our previous study showed higher uncertainty underestimation in older compared to younger adults (Nassar et al., 2016), we directly compared younger and older adults using a Mann-Whitney $\mathrm{U}$ test. This test yielded significantly lower uncertainty underestimation in younger adults compared to older adults $(u=394.0, p=0.038)$. That is, in line with our previous work the results suggest reduced uncertainty-driven learning in older adults. We further investigate the relationship between the current and previous result below (see Control model).

The surprise-sensitivity parameter indicates the influence of changepoint probability on the learning rate. Lower surprise sensitivity reflects lower learning rates in the range of large prediction errors that are most likely due to changepoints (Fig. SM 2b). The surprise-sensitivity estimates shown in Fig. SM 2e suggest that all age groups considered changepoint probability, albeit not as much as the reduced Bayesian model, which uses a surprise sensitivity equal to 1 . Moreover, the ANOVA $(H=4.363, p=0.225)$ did not indicate significantly different median surprise-sensitivity parameters between children (median $=0.153$, IQR 0.053 to 0.566 ), adolescents (median $=0.206$, IQR 0.121 to 0.288 ), younger adults (median $=0.283$, IQR 0.179 to 0.43 ), and older adults (median $=0.24$, IQR 0.103 to 0.354 ).

The hazard-rate parameter models assumptions about the frequency of changepoints. A higher hazard rate reflects the assumption that changepoints occur more frequently in the task and leads to higher levels of changepoint probability and thus higher learning rates for small and intermediate prediction errors (Fig. SM 2c). The true but unknown hazard rate was approximately 0.1, i.e., the hazard-rate parameter estimates shown in Fig. SM $2 \mathrm{f}$ suggest that most participants overestimated the frequency of changepoints in the task. However, the ANOVA $(H=2.715, p=0.438)$ did not yield significant hazard-rate parameter differences between children (median $=0.461$, IQR 0.28 to 0.545 ), adolescents (median $=0.504$, IQR 0.354 to 0.616 ), younger adults (median $=0.45$, IQR 0.291 to 0.595 ), and older adults (median $=0.495$, IQR 0.428 to 0.571$)$. 

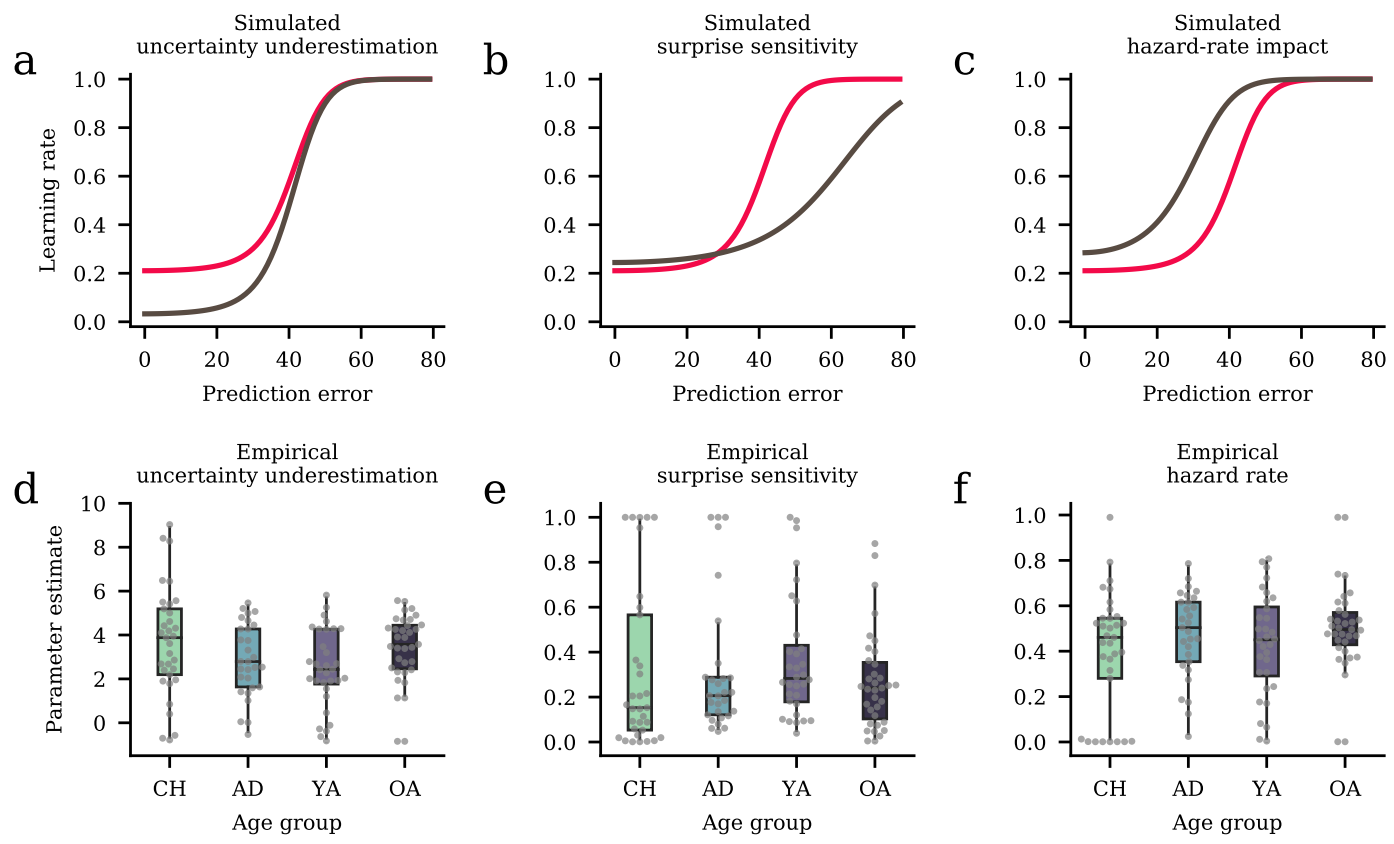

Figure SM 2. Hypothetical and estimated learning deficits resulting from specific deviations from the reduced Bayesian learning model. a-c) The reduced Bayesian model (red line) uses a high learning rate in response to large prediction errors that are more likely associated with changepoints. In the range of small prediction errors that are more likely due to variability, it uses a lower learning rate to average out the variability in the outcomes. We hypothesized that learning deficits may stem from specific deviations from the learning-rate computations of the reduced Bayesian model, including a) an underestimation of uncertainty, b) lower surprise sensitivity, and c) a subjectively high hazard rate (dark lines). a) An underestimation of uncertainty leads to a specific learning deficit in the range of small prediction errors unlikely to be changepoints. That is, the model has a reduced ability to average out the variability in the outcomes. b) Insensitivity to surprise leads to a learning deficit in the range of large prediction errors that are likely to be changepoints. Consequently, the model has a reduced ability to respond to changepoints appropriately. c) The hazard rate reflects the model's assumed frequency of changepoints. A subjectively higher hazard rate, i.e., an overestimation of how often changepoints occur, is associated with higher learning rates in the range of small and intermediate prediction errors. d-f) Estimated parameters reflecting d) uncertainty underestimation, e) surprise sensitivity, and f) subjective hazard rate for children (CH), adolescents $(\mathrm{AD})$, younger adults (YA), and older adults (OA). 
Here we also present the additionally estimated parameters of the mixture model. The motor-noise parameter $o_{0}$ modeled prediction noise that was independent of the update magnitude (Fig. SM 3a). An ANOVA did not yield a significant difference between children (median $=3.859$, IQR 1.463 to 5.513), adolescents (median $=4.251$, IQR 2.099 to 6.201), younger adults (median $=2.232$, IQR 1.246 to 4.197 ), and older adults (median $=3.498$, IQR 2.241 to 4.931$)(H=7.079, p=0.069)$.

The learning-rate noise parameter $o_{1}$ modeled prediction noise depending on the update magnitude (Fig. SM 3b). An ANOVA indicated significant differences between the age groups $(H=10.364, p=$ 0.016). Mann-Whitney $U$ tests yielded a significant difference between children (median $=0.189$, IQR 0.124 to 0.306 ) and adolescents (median $=0.146$, IQR 0.091 to $0.209 ; u=628.0, p=0.036$ ), adolescents and younger adults (median $=0.226$, IQR 0.159 to $0.372 ; u=270.0, p=0.005$ ), and between younger and older adults (median $=0.155$, IQR 0.098 to $0.249 ; u=738.0, p=0.026$ ). The test did not show a significant difference between children and younger adults $(u=449.0, p=0.303)$, children and older adults $(u=698.0, p=0.141)$, and between adolescents and older adults $(u=464.0, p=0.562)$.

The intercept parameter $b_{0}$ of the logistic function was indicative of perseveration (Fig. SM 3c). An ANOVA yielded significant median intercept differences between the age groups $(H=21.702, p<0.001)$. Post-hoc tests indicated a significant difference between children (median $=8.263$, IQR 1.522 to 20.373) and adolescents (median $=0.703$, IQR -12.633 to $4.587 ; u=706.0, p=0.001$ ), children and younger adults (median $=1.002, \mathrm{IQR}-7.081$ to $3.717 ; u=780.0, p=0.001)$, adolescents and older adults (median $=$ 7.585, IQR 4.141 to $12.153 ; u=276.0, p=0.002)$, younger adults and older adults $(u=289.0, p=0.001)$ but not between children and older adults $(u=640.0, p=0.446)$ and adolescents and younger adults $(u$ $=461.0, p=0.971)$.

Perseveration was also reflected in the slope parameter $b_{1}$ of the logistic function (Fig. SM 3d). An ANOVA did not yield significant median slope differences between the age groups $(H=4.526, p=0.21)$ (children: median $=-0.17$, IQR -0.25 to -0.084 ; adolescents: median $=-0.215$, IQR -0.309 to -0.125 ; younger adults: median $=-0.253$, IQR -0.396 to -0.131 ; older adults: median $=-0.186$, IQR -0.31 to -0.095$)$. The combination of the intercept and slope parameters are reflected in higher perseveration probabilities in children and older adults, which are especially evident in the range of small and intermediate updates (Figure 2d).

The reward-bias parameter $q$ modeled the influence of high versus low reward on the learning rate (Fig. SM 3e) (children: median $=0.032$, IQR -0.005 to 0.077 ; adolescents: median $=0.059$, IQR -0.01 to 0.113 ; younger adults: median $=0.015$, IQR -0.004 to 0.061 ; older adults: median $=0.026$, IQR -0.027 to 0.061). An ANOVA did not yield a significant difference between the age groups $(H=4.424, p=0.219)$.

Finally, the catch-trial parameter $\sigma_{H}$ modeled the influence of catch trials on the update magnitude (Fig. SM 3f) (children: median $=10.899$, IQR 6.513 to 21.603 ; adolescents: median $=9.507$, IQR 4.35 to 21.925; younger adults: median $=14.138$, IQR 9.042 to 19.696 ; older adults: median $=15.269$, IQR 4.938 to 30.642$)$. An ANOVA did not yield significant differences between the age groups $(H=1.035, p$ $=0.793)$. 

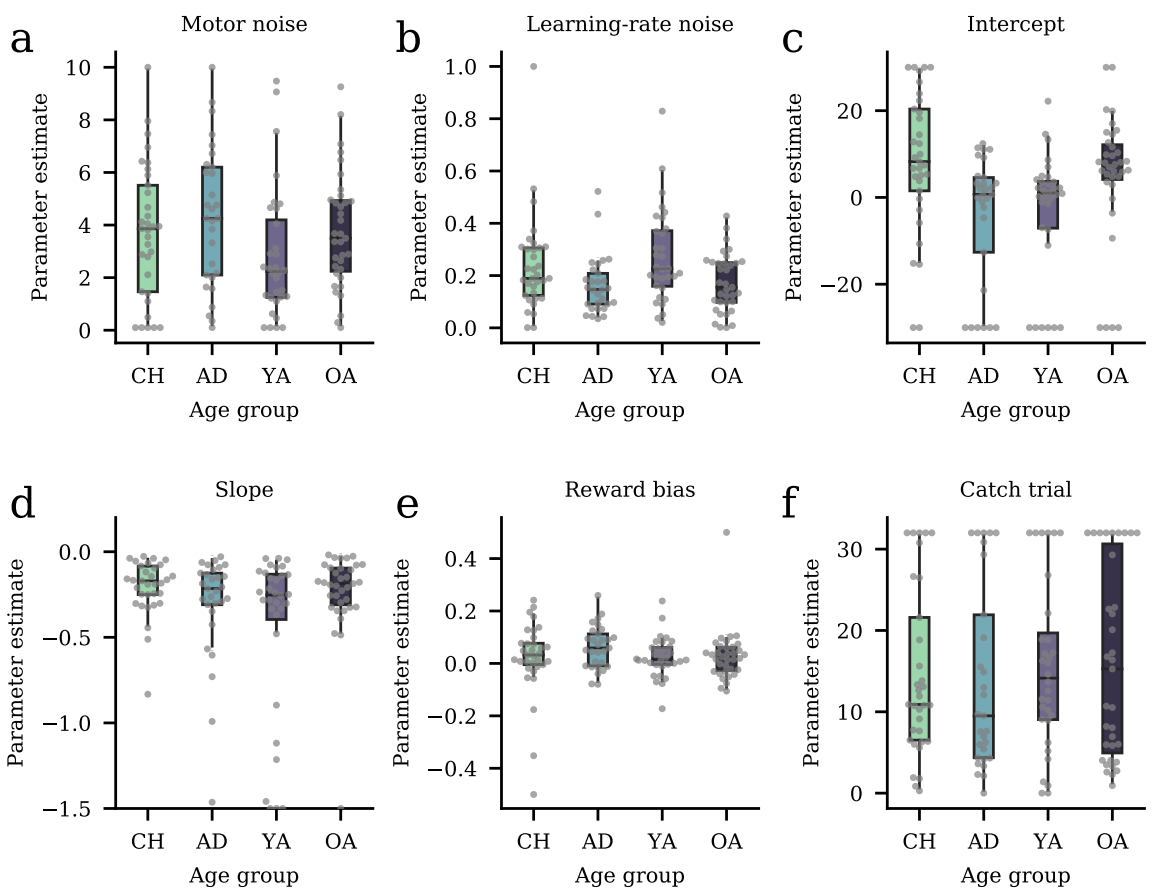

Figure SM 3. Additional model-parameter estimates (first experiment). a) Motor noise. b) Learning-rate noise. c) Intercept of the logistic function. d) Slope of the logistic function. e) Reward-bias parameter. f) Catch-trial parameter. 


\section{Control model}

A direct comparison of uncertainty underestimation revealed a significant difference between younger and older adults (Fig. SM 2d). This result should be interpreted with caution because the ANOVA comparing uncertainty underestimation across all groups did not yield a significant difference. Moreover, as described in Evaluation and estimation, we used multiple random starting points to estimate the free parameters of our models. All reported analyses are conditional on a fixed random seed, but we observed that the test outcomes of uncertainty underestimation were affected by the seed in that the $p$-values could sometimes favor the hypothesis of differences in uncertainty underestimation on the group level but also sometimes reject it. The effect of uncertainty underestimation seems to be considerably weaker than in Nassar et al. (2016), which could be related to the additional perseveration component in our model. To examine if the age-related differences in uncertainty underestimation might be more clearly pronounced in a model without perseveration, we additionally inspected uncertainty underestimation in our control model. In Fig. SM 4 we show the parameter estimates of the control model without perseveration, which fit the data worse compared to the mixture model that captured perseveration (see Model comparison). To test if the uncertainty-underestimation parameters in this model were different between the age groups, we also applied an ANOVA $(H=17.545, p=0.001)$. Post-hoc tests showed a significant difference between children $($ median $=4.604$, IQR 2.901 to 6.48$)$ and adolescents $($ median $=2.456$, IQR 2.012 to $3.728 ; u=$ $710.0, p=0.001$ ), children and younger adults (median $=2.97$, IQR 1.928 to $4.027 ; u=780.0, p=0.001$ ), adolescents and older adults (median $=3.732$, IQR 2.663 to $5.405 ; u=317.0, p=0.01$ ), and younger and older adults $(u=381.0, p=0.025)$. The test did not yield a significant difference between children and older adults $(u=683.0, p=0.198)$, and adolescents and younger adults $(u=444.0, p=0.778)$. These results suggest that the perseveration component of our best-fitting model partly captures variance that the uncertainty-underestimation parameter captures in the control model without perseveration. We think this explanation is reasonable because both uncertainty underestimation and perseveration lead to an under-adjustment of predictions. Uncertainty underestimation leads to an update that is smaller than prescribed by the normative version of the reduced Bayesian model, while perseveration leads to the entire absence of an update. Moreover, both effects occur primarily after small prediction errors. Our previous and current results are thus in line with each other; however, the interpretation of why older adults used lower average learning rates in the range of small prediction errors is different. The current study favors the interpretation that older adults (and children) primarily use lower learning rates because they often perseverate. On some trials, they update their predictions, while on other trials, they refrain from updating. This point demonstrates that psychological interpretations drawn contingent on selected model parameters strongly depend on the tested model space (Nassar and Frank, 2016). 

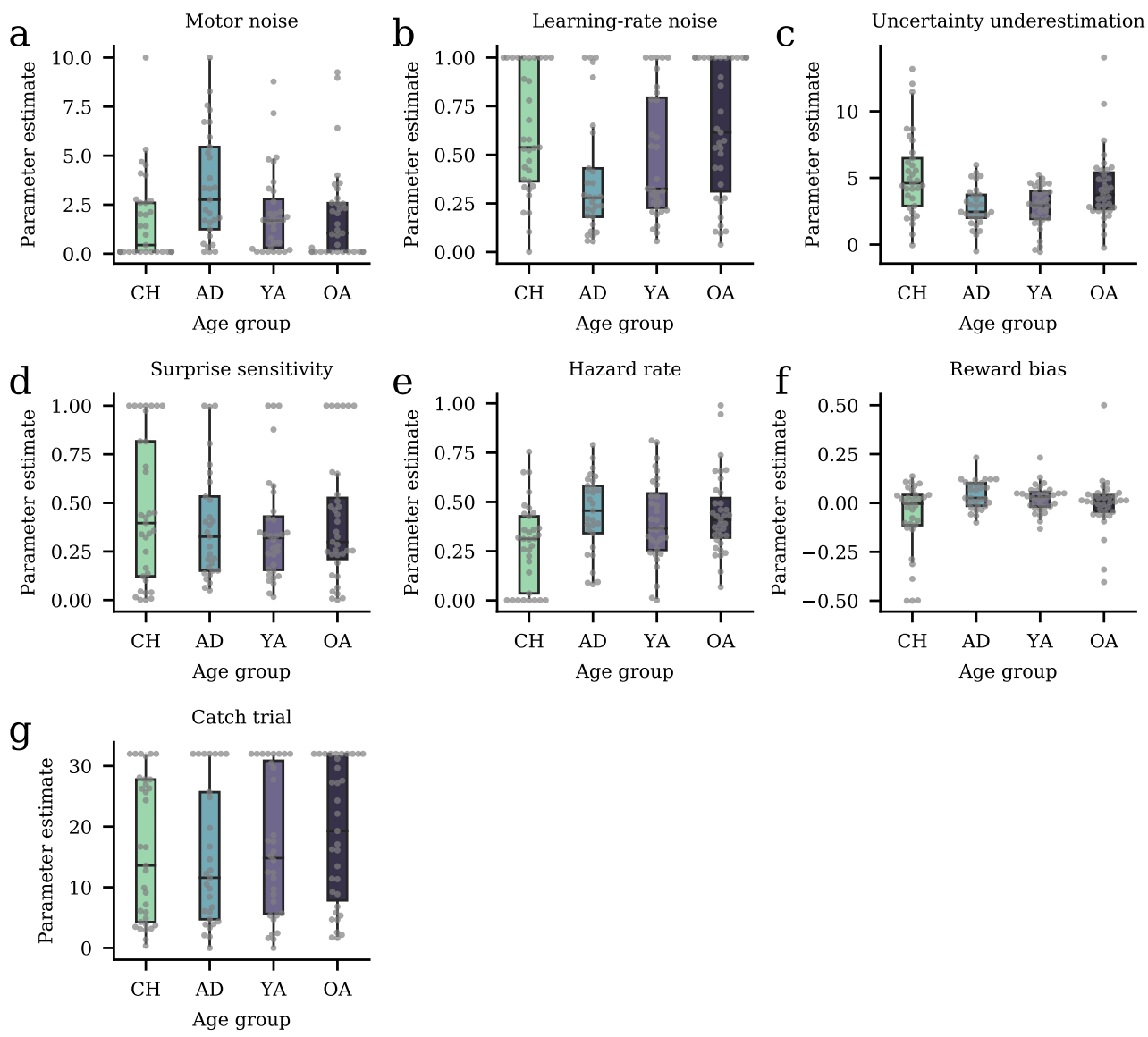

Figure SM 4. Model parameter estimates of the control model (first experiment). a) Motor noise. b) Learningrate noise. c) Uncertainty underestimation. d) Surprise sensitivity. e) Hazard rate. f) Reward-bias parameter. g) Catch-trial parameter. 


\section{Model comparison}

To test if the mixture model between the reduced Bayesian model and the perseveration model describes the data better than the control model without perseveration, we compared the cumulated Bayesian information criterion (BIC) (Stephan et al., 2009) and the corresponding protected exceedance probabilities (Rigoux et al., 2014). The BIC for each participant was defined as

$$
\mathrm{BIC}:=\ell-\frac{k}{2} \ln (T)
$$

where $\ell$ denotes the maximum log-likelihood, $k$ the number of free parameters and $T$ the number of trials and where higher values indicate a better model fit (Fig. SM 5a). We used protected exceedance probabilities for model comparison on the group level, which indicates the probability that a particular model is more likely than any other model of the model space (Fig. SM 5b). Together, this indicated that in each age group, the mixture model fits the data better than the reduced Bayesian model without perseveration. We finally counted the number of participants, in which the mixture model fit the data better than the reduced Bayesian model without perseveration. This indicated that in 124 out of 129 participants, the model comparison favored the mixture model.

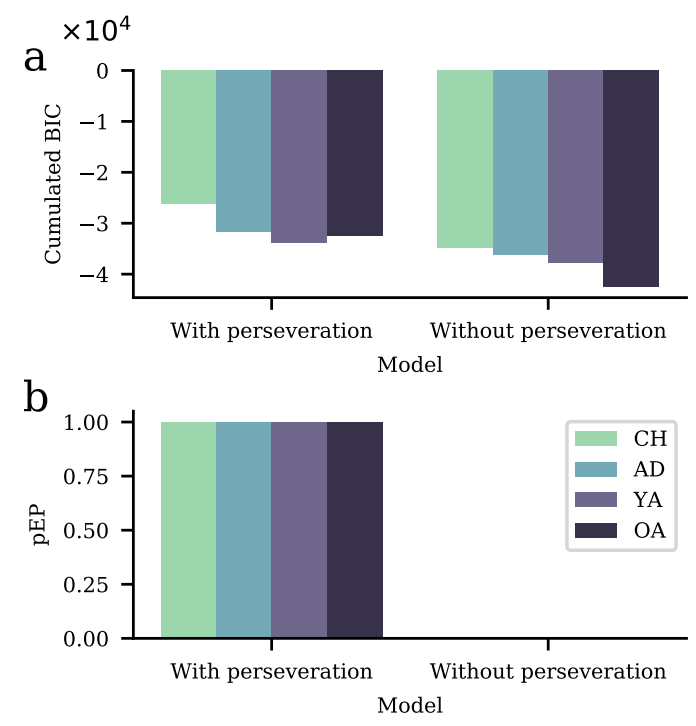

Figure SM 5. Model comparison. a) Cumulated Bayesian information criterion (BIC) of the reduced Bayesian model with perseveration and the reduced Bayesian model without perseveration, plotted separately for each age group. Higher BIC values indicate a better model fit, that is, in all age groups, the reduced Bayesian model with perseveration fit the data better than the model without perseveration. b) This is supported by the larger protected exceedance probabilities (pEP, all $>0.99$ ) of the model with perseveration. 


\section{Results follow-up experiment}

According to Wilcoxon sign-rank tests, no age group showed a significant perseveration-probability difference between the high- and low-reward condition (children: median $=0.002$, IQR -0.029 to 0.028 , $w=231.0, p=0.739$; younger adults: median $=0.004$, IQR -0.018 to $0.018, w=148.0, p=0.696$; older adults: median $=0.001$, IQR -0.014 to $0.025, w=273.0, p=0.893$ ) (Fig. SM 6a). It is unclear why we did not obtain a significant perseveration difference between the two conditions. It is possible that the incentive manipulation failed to increase the motivation to reach a higher learning performance, potentially because the reward cues were not sufficiently salient or the differences in the reward magnitudes were not kept in mind anymore after the instructions. The absence of an effect of the manipulation on perseveration should not be interpreted as evidence for the absence of this relationship, and other incentive manipulations may affect perseveration.

Because our primary interest in the follow-up experiment was to explore which task manipulations are associated with decreased perseveration, we did not further analyze the difference between high- and low-incentive trials but focused on the difference between the shifting- and stable-bucket condition. For the sake of completeness, we here provide the age-related estimation-error differences between the highand low-reward trials (Fig. SM 6b). In all age groups, Wilcoxon sign-rank tests yielded a significant estimation-error difference between the high- and low-reward condition (children: median $=1.188$, IQR 0.293 to $2.423, w=59.0, p<0.001$; younger adults: median $=1.291$, IQR 0.981 to $2.144, w=24.0$, $p<0.001$; older adults: median $=1.062$, IQR 0.512 to $2.021, w=37.0, p<0.001)$.

We next provide additional details on the differences in the estimated perseveration probabilities in the shifting- compared to the stable-bucket environment. According to Wilcoxon sign-rank tests, children (median $=-0.455$, IQR -0.602 to $-0.348, w=0.0, p<0.001$ ), younger adults (median $=-0.21$, IQR -0.385 to $-0.16, w=0.0, p<0.001$ ), and older adults (median $=-0.488$, IQR -0.58 to $-0.376, w=0.0$, $p<0.001)$ perseverated significantly less in the shifting-bucket environment compared to the stable-bucket environment (Fig. SM 6c).

Moreover, children (median $=3.671$, IQR 0.631 to $4.785, w=71.0, p=0.001$ ) and older adults (median $=3.636$, IQR 1.316 to $8.234, w=42.0, p<0.001$ ) showed higher estimation errors in the shifting-compared with the stable-bucket environment. In younger adults, this test did not yield a significant estimation-error difference between the environments (median $=0.097$, IQR -0.776 to $1.103, w=139.0, p=0.527$ ) (Fig. SM 6d).

Finally, we zoomed into the perseveration trials in the shifting-bucket condition by dividing the trials in this condition into "edge" and regular trials. Edge trials reflect trials in which the starting position of the bucket was either $z_{t}=0$ (left edge of the screen) or $z_{t}=300$ (right edge of the screen). In these trials, the bucket-shift effect could be different from regular trials because the shift in the location could not affect the starting position beyond the edge. As shown in Fig. SM 6e, perseveration was practically absent in regular trials and thus primarily occurred during edge trials, suggesting that the few cases of perseveration (c.f. Fig. SM 6c, shifting-bucket condition) were driven by edge trials in which the bucket-shift effect was truncated. A similar result was observed for motor-perseveration trials (Fig. SM 6f). Motor perseveration refers to the prediction at the default bucket location (see Data preprocessing), which was absent in regular trials but occasionally took place during edge trials. Together, these results support the conclusion that our manipulation strongly reduced the perseverative behavior of our participants. 

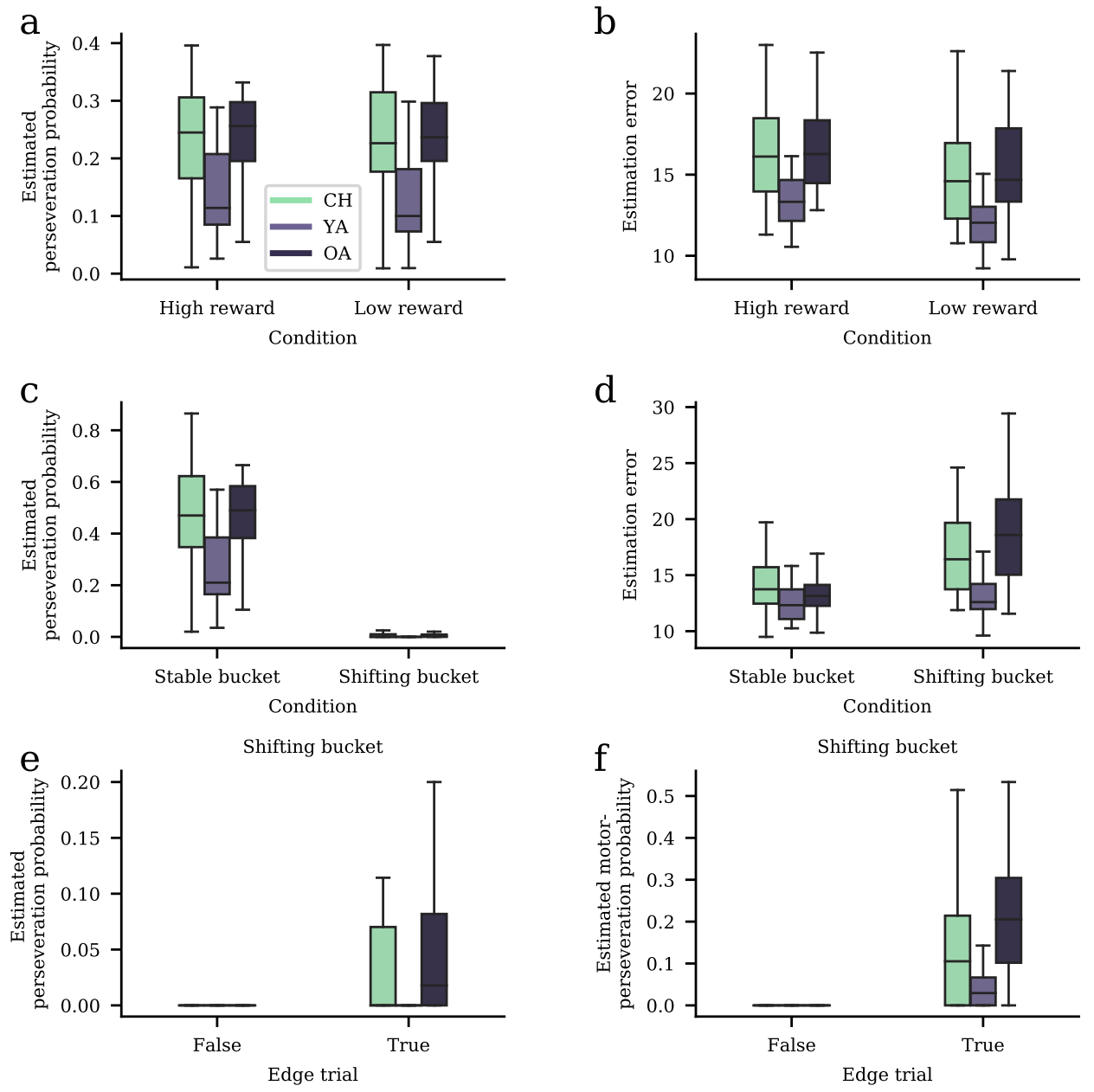

Figure SM 6. Supplementary results of the follow-up experiment. a) Estimated perseveration probability in the reward conditions. b) Estimation errors in the reward conditions. c) Estimated perseveration probability in the bucket conditions. d) Estimation error in the the bucket conditions. e) Estimated perseveration probability plotted separately for regular and edge trials. f) Estimated motor-perseveration probability plotted separately for regular and edge trials. 


\section{Average learning rate follow-up experiment}

In the follow-up experiment, we tested which factors determined participants' belief updates. Similar to the first experiment, we analyzed average learning rates across all trials, indicating to which extent prediction errors influenced the belief update on average (Fig. 4e). The ANOVA showed that learning rates were significantly different between the age groups $(H=21.951, p<0.001)$. The post-hoc tests indicated significantly lower learning rates in children (median $=0.729$, IQR 0.65 to 0.838 ) compared to younger adults (median $=0.884$, IQR 0.86 to $0.923 ; u=131.0, p<0.001$ ) but not compared to older adults (median $=0.771$, IQR 0.699 to $0.854 ; u=466.0, p=0.427$ ). Similarly, younger adults had significantly higher learning rates compared to older adults $(u=683.0, p<0.001)$. As in the first experiment, these results show that children and older adults use lower learning rates than younger adults, i.e., they use prediction errors to a lower degree for updating their beliefs about the helicopter.

\section{Computational modeling follow-up experiment}

\section{Mixture model}

To confirm that age-related differences in the incorporation of random task factors capture performance differences beyond the computational factors of our model, we extended the model with a bias parameter. As illustrated in Fig. 4b, in this extended version, the model's prediction of the next outcome was biased towards the shifted bucket position. This bias was governed by an additional free parameter, where higher parameter values indicate a stronger bias (Fig. 4g). According to an ANOVA, the parameters were significantly different between the age groups $(H=24.251, p<0.001)$. Post-hoc tests indicated a significant difference between children (median $=0.158$, IQR 0.065 to 0.277 ) and younger adults (median $=0.052$, IQR 0.02 to $0.086 ; u=567.0, p=0.003$ ), children and older adults (median $=0.302$, IQR 0.133 to $0.434 ; u=323.0, p=0.008)$, and between younger and older adults $(u=127.0, p<0.001)$. Together, these findings confirm our descriptive results (Fig. 4f) that the shifted bucket differently biased belief updating across the lifespan.

Moreover, an ANOVA did not yield a significantly different motor-noise parameter $o_{0}$ between children (median $=7.327$, IQR 5.927 to 9.523$)$, younger adults (median $=5.853$, IQR 3.874 to 7.05 ), and older adults (median $=6.614$, IQR 4.707 to 8.559$)(H=5.823, p=0.054)($ Fig. SM 7a).

An ANOVA yielded a significantly different learning-rate noise parameter $o_{1}$ between the age groups $(H=10.123, p=0.006)$. Post-hoc tests tests showed a significant difference between children (median $=0.209$, IQR 0.107 to 0.314 ) and younger adults (median $=0.096$, IQR 0.067 to $0.163 ; u=549.5, p=$ 0.008 ) and between younger adults and older adults (median $=0.21$, IQR 0.14 to $0.279 ; u=234.0, p=$ $0.003)$. The test did not indicate a significant difference between children and older adults $(u=544.0, p$ $=0.828$ ) (Fig. SM 7b).

The ANOVA also yielded a significant age-related difference in the intercept parameter $b_{0}$ of the logistic function $(H=10.827, p=0.004)$. Follow-up tests indicated a significant difference between children $($ median $=7.943$, IQR 5.656 to 11.863 ) and younger adults (median $=3.16$, IQR 0.76 to 7.486 ; $u=567.0, p=0.003$ ) and between younger and older adults (median $=6.563$, IQR 4.285 to $8.79 ; u=$ 250.0, $p=0.007)$. The test did not indicate a significant difference between children and older adults $(u$ $=600.0, p=0.341$ ) (Fig. SM 7c).

With respect to the slope of the logistic function $b_{1}$, an ANOVA showed significant differences between the age groups $(H=6.531, p=0.038)$. Children (median $=-0.319$, IQR -0.467 to -0.208$)$ had significantly higher slopes than older adults (median $=-0.535$, IQR -0.813 to $-0.331 ; u=684.0, p=0.04$ ). Younger adults (median $=-0.309$, IQR -0.407 to -0.226$)$ had significantly higher slopes than older adults $(u=$ 
575.0, $p=0.022)$. Finally, the slopes of children and younger adults were not significantly different $(u=$ 403.0, $p=0.805$ ) (Fig. SM 7d).

For the uncertainty-underestimation parameter $u$, an ANOVA did not indicate significant differences (children: median $=3.092$, IQR 2.42 to 4.49; younger adults: median $=2.569$, IQR 1.002 to 3.914; older adults: median $=3.622$, IQR 2.289 to 4.854$)$ between the age groups $(H=4.562, p=0.102)$ (Fig. SM $7 \mathrm{e})$.

Moreover, the test yielded a significant difference in the surprise-sensitivity parameter $s(H=9.667$, $p=0.008$ ). Post-hoc rank-sum tests indicated a significant difference between children (median $=0.079$, IQR 0.023 to 0.351 ) and older adults (median $=0.465$, IQR 0.089 to $0.639 ; u=321.0, p=0.007$ ) and between younger adults (median $=0.098$, IQR 0.058 to 0.2$)$ and older adults $(u=263.0, p=0.013)$. The test did not yield a significant difference between children and younger adults $(u=339.0, p=0.429)$ (Fig. SM 7f).

Next, the test yielded a significant age-related difference in the hazard-rate parameter $h(H=6.521, p$ $=0.038)$ (Fig. SM 7g). Children (median $=0.527$, IQR 0.446 to 0.552 ) had significantly lower hazard-rate parameters compared to younger adults (median $=0.628$, IQR 0.464 to $0.718 ; u=260.0, p=0.036$ ) but not compared to older adults (median $=0.52$, IQR 0.43 to $0.584 ; u=542.0, p=0.849$ ). Younger adults had significantly higher hazard-rate parameters compared to older adults $(u=580.0, p=0.018)$.

Finally, the ANOVA did not yield a significant age-related difference in the catch-trial parameter $\sigma_{H}$ $(H=0.929, p=0.628)$ between children (median $=20.158$, IQR 9.832 to 32.0 ), younger adults (median $=15.714$, IQR 6.583 to 32.0) and older adults (median $=30.917$, IQR 5.287 to 32.0) (Fig. SM 7h). 

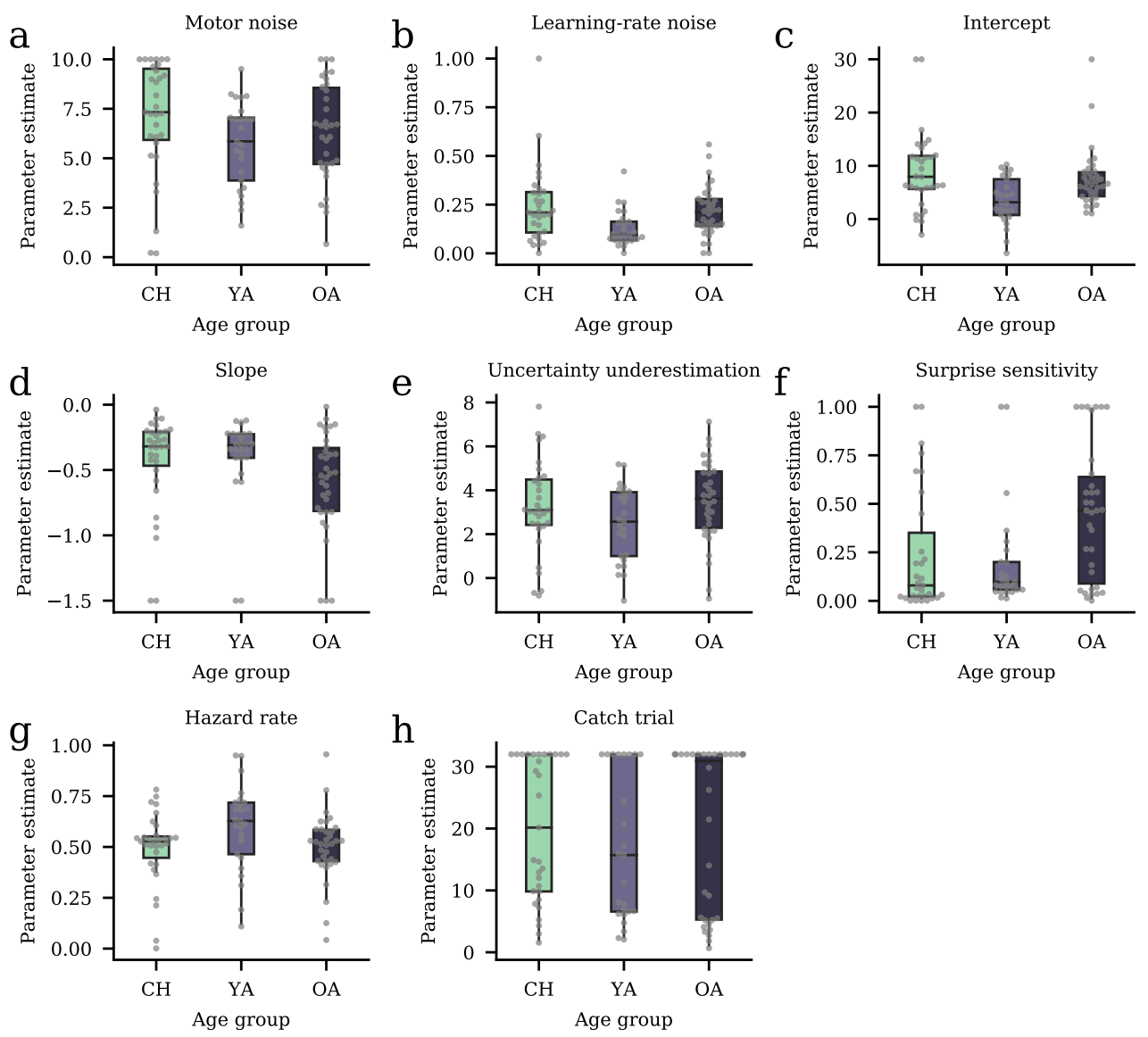

Figure SM 7. Additional model-parameter estimates (follow-up experiment). a) Motor noise. b) Learning-rate noise. c) Intercept of the logistic function. d) Slope of the logistic function. e) Uncertainty underestimation. e) Surprise sensitivity. f) Hazard rate. h) Catch-trial parameter. 


\section{Parameter-recovery analysis}

To test if the parameters of the mixture model between the reduced Bayesian model and the perseveration model can accurately be estimated, we conducted a parameter-recovery study. To examine if a broad range of parameter combinations are recoverable, we used randomly selected parameters during the simulations. These parameters were based on the participants' empirical parameter distributions (estimated using the seaborn distplot function) and additionally constrained between the approximate minimum and maximum empirical parameter estimates. The parameter-recovery results for the first (Fig. SM 8) and follow-up experiment (Fig. SM 9) suggest that parameters can sufficiently be estimated except for the catch-trial parameter, which was often estimated at the upper boundary. This result indicates that the empirical results regarding catch trials should be cautiously treated.
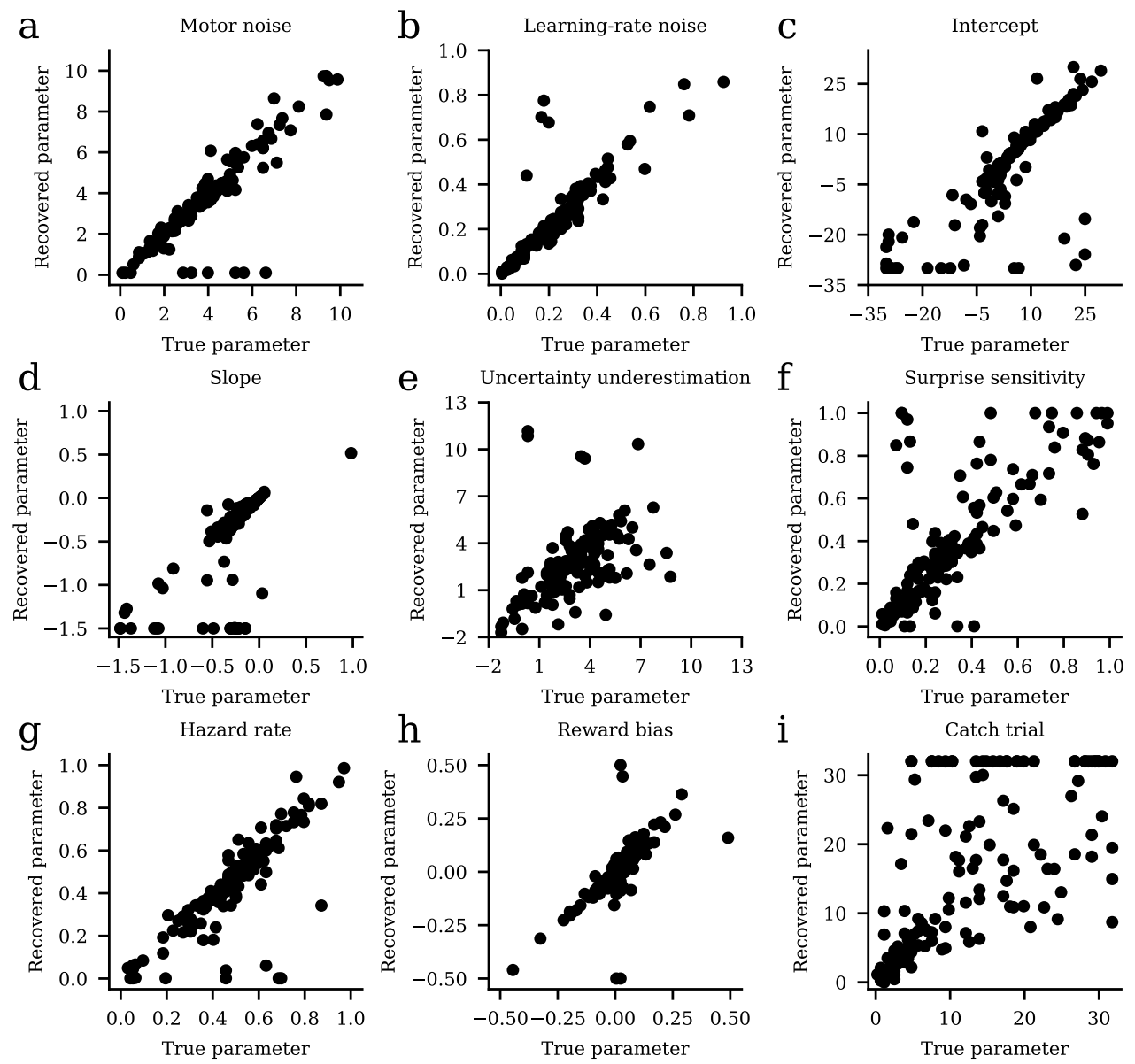

Figure SM 8. Parameter recovery first experiment. a) Motor noise. b) Learning-rate noise. c) Intercept of the logistic function. d) Slope of the logistic function. e) Uncertainty underestimation. f) Surprise sensitivity. g) Hazard rate. h) Reward-bias parameter. i) Catch-trial parameter. 

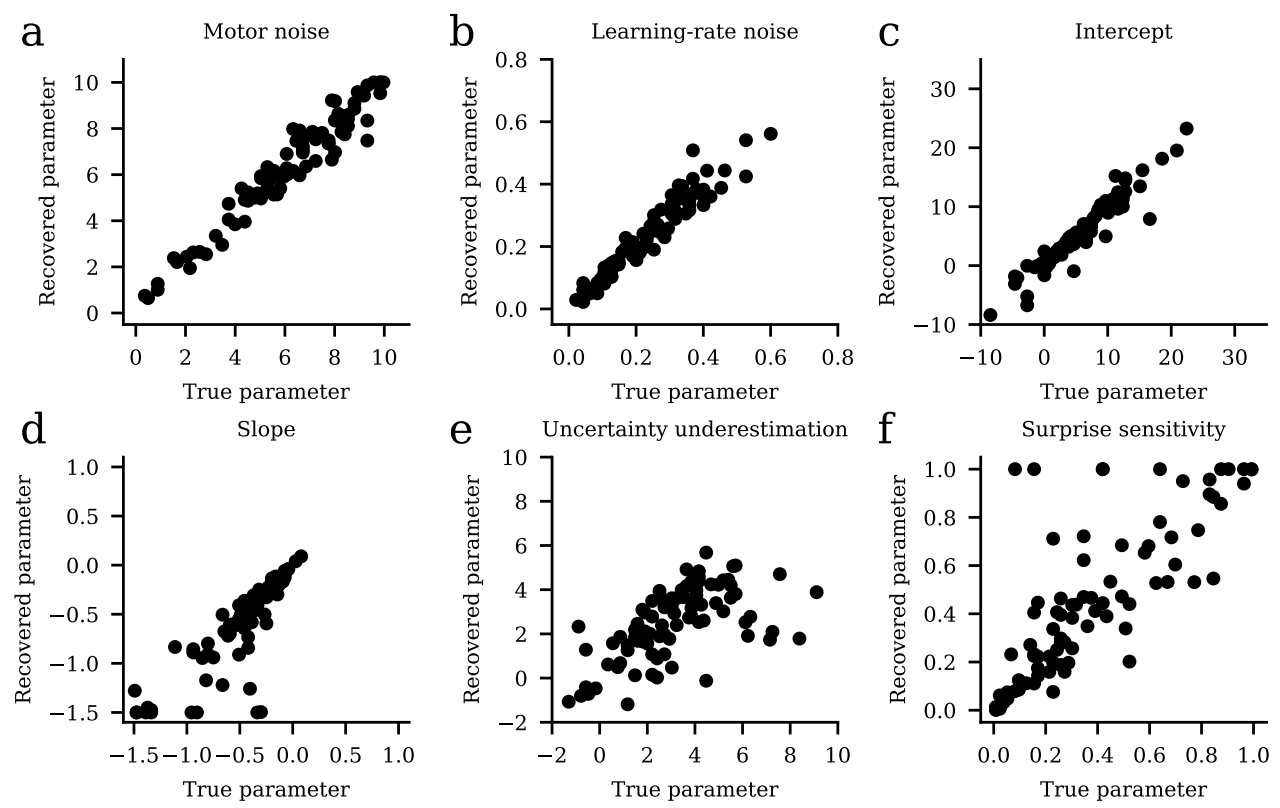

h
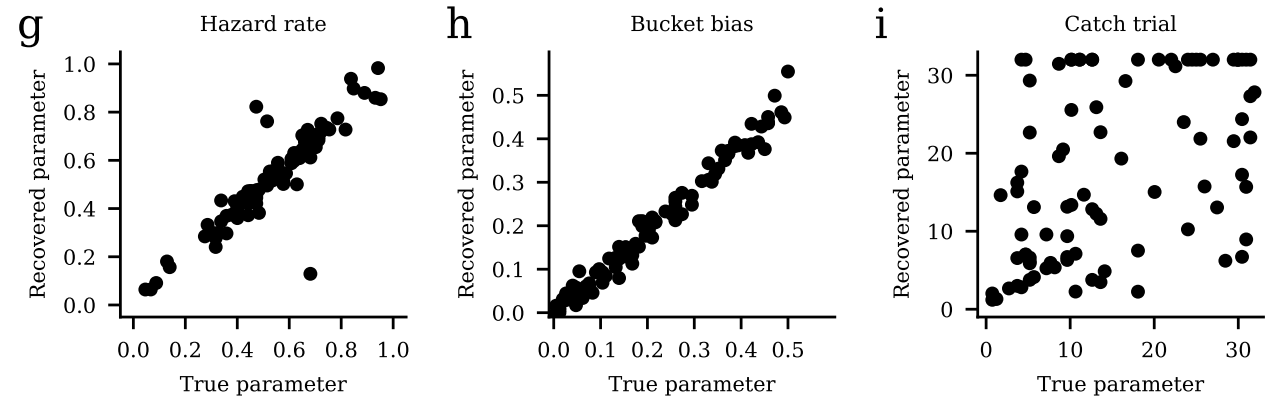

Figure SM 9. Parameter recovery follow-up experiment. a) Motor noise. b) Learning-rate noise. c) Intercept of the logistic function. d) Slope of the logistic function. e) Uncertainty underestimation. f) Surprise sensitivity. g) Hazard rate. h) Belief-updating bias. i) Catch-trial parameter. 


\section{Model validation}

\section{First experiment}

One potential concern with using computational models as data-analytic tools is that although they make quantitative predictions of the data, they might fail to reproduce the observed data in simulations qualitatively (Palminteri et al., 2017). To test if our model-based analyses accurately captured participants' behavior, we used the estimated mixture model to simulate learning behavior. First, to test if the estimated reduced Bayesian model with an additional perseveration component qualitatively reproduces participant behavior, we applied it to the same sequence of data as experienced by our participants. To obtain smoother simulation results, we repeated this procedure across three cycles. The comparison between empirical and simulated data is shown in Fig. SM 10a (perseveration probability) and Fig. SM 10b (estimation errors). In addition, as shown in Fig. SM 10c,d, we also used simulations to explore if the reduced Bayesian model without perseveration would predict similar performance levels between the age groups. Here we conducted the simulations as described above but adjusted the parameters such that perseveration probability was approximately equal to zero. These results suggested similar performance levels and motivated our followup experiment that tested if age-related performance differences can be eliminated with manipulations to reduce perseveration.

a

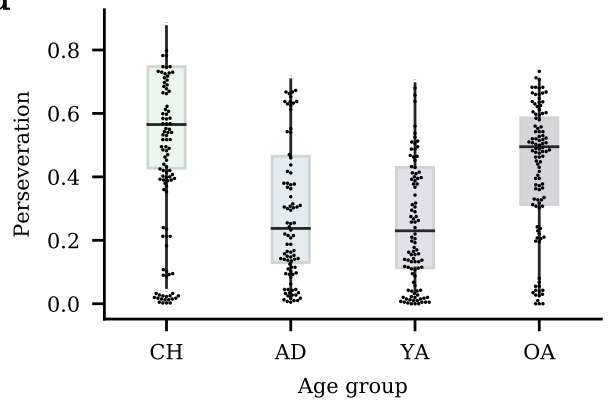

Boxplots: participants | Points: simulations

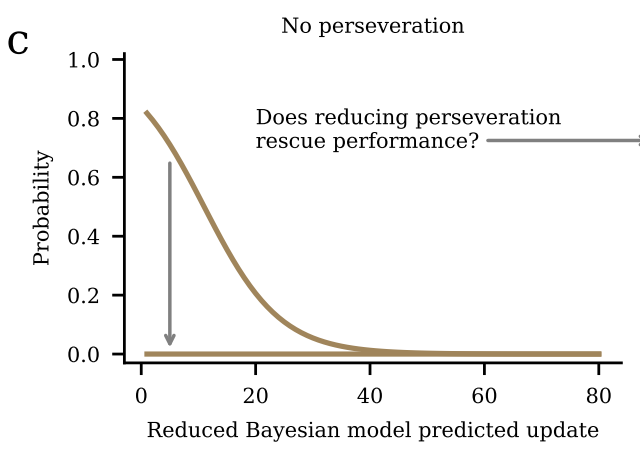

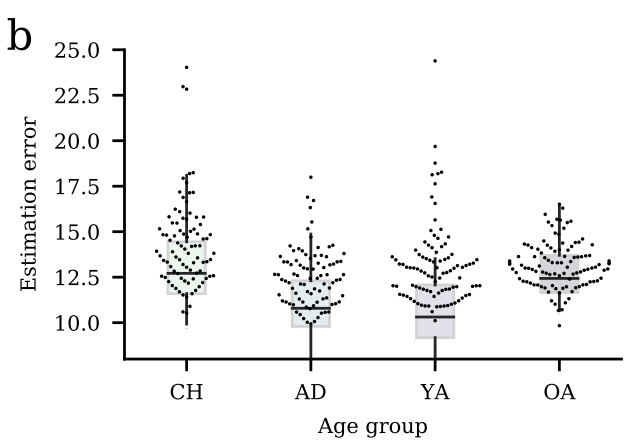

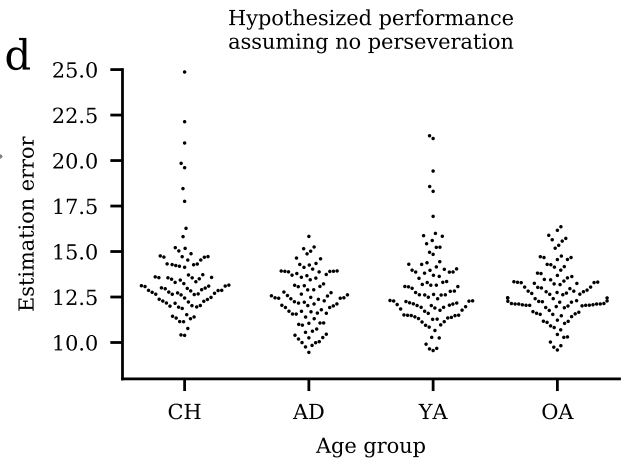

Figure SM 10. Simulation results. a-b) To test whether the mixture model can capture age differences in perseveration and estimation errors, we simulated learning behavior for each age group. Each dot represents a simulated participant, and the boxplots show the empirical data for comparison. The results suggest that the mixture model qualitatively captures the pattern of a) perseveration and b) estimation errors across the age groups. c) To test whether perseveration might contribute to age-related performance differences, we simulated learning behavior from models best fit to each subject in all four age groups but set the perseveration probabilities to zero in all cases. d) The simulation results suggest that, without perseveration, the four age groups would perform at similar levels. 


\section{Follow-up experiment}

As in our model-validation analyses of the first experiment, we conducted the simulations with the estimated parameters and the same outcomes observed by our participants. These simulations revealed that the simulated perseveration (Fig. SM 5a) and estimation-error (Fig. SM 5b) differences qualitatively matched our empirically obtained results, which suggests that our computational model accurately captured participants' behavior.
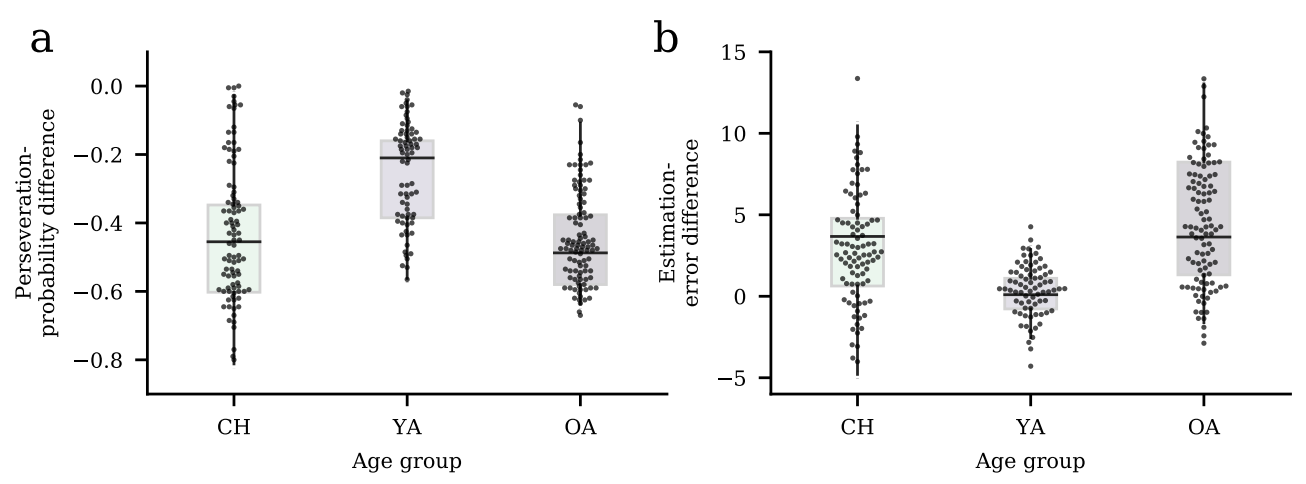

Boxplots: participants | Points: simulations

Figure SM 11. Model validation follow-up experiment. To test if the reduced Bayesian model with the additional belief-updating bias qualitatively captures the difference in perseveration and estimation errors between the shifting- and stable-bucket environment, we used the model to simulate behavior across three cycles, where each point in the plot represents one simulation. a). Comparison of the simulated and empirical perseveration probability. b) Comparison of the simulated and empirical estimation errors. 


\section{Robust regression with age group}

We also applied a robust linear regression model with age group

$$
d_{i}=\beta_{0}+\beta_{1} \hat{p}(\text { pers })_{i}+\beta_{2}^{y a} g_{i}+\beta_{2}^{c h} g_{i}+\beta_{3}^{y a} \hat{p}(\text { pers }) g_{i}+\beta_{3}^{c h} \hat{p}(\text { pers }) g_{i}
$$

where $g_{i}$ denotes age group that we coded as dummy variable with older adults as reference category. The model thus contained an additional main effect of age group with regression weight $\beta_{2}$ and an interaction between age group and perseveration probability with regression weight $\beta_{3}$.

This model revealed a significant main effect of perseveration probability $\beta_{1}=0.8404(z=5.085, p<$ 0.001 ), and a significant interaction between perseveration probability and the level of younger adults of the age group variable $\beta_{3}^{y a}=-0.8044(z=-3.377, p=0.001)$ and the level of children $\beta_{3}^{\text {ch }}=$ $-0.5699(z=-2.858, p=0.004)$. All other parameters were not significant: $\beta_{0}=-0.1232(z=$ $-1.510, p=0.131), \beta_{2}^{y a}=0.1732(z=1.780, p=0.075), \beta_{2}^{c h}=0.1681(z=1.699, p=0.089)$. Hence, this robust regression with age group suggests that the bucket-shift parameter is systematically related to perseveration and that this relationship is weaker in younger adults and children compared to older adults. For the predicted values according to this model, see Fig. SM 12.

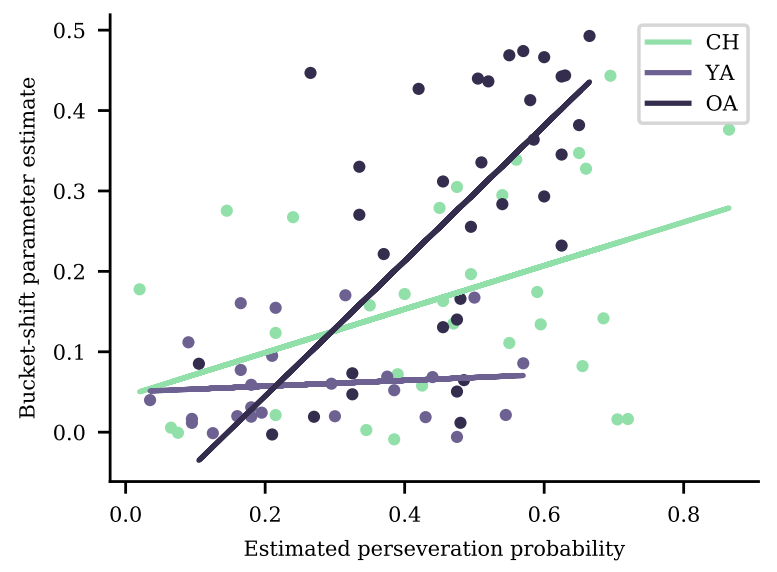

Figure SM 12. Robust linear regression with age group. 


\section{Satisficing model}

\section{Percent-point function}

The percent-point function is the inverse of the Gaussian cumulative distribution function $F(x)$,

$$
Q(p)=\inf \{x \in \mathbb{R}: p \leq F(x)\},
$$

where $p$ denotes a probability $\in[0,1]$. This function returns the minimum $x$ of the values where $F(x)$ exceeds probability $p$. For an example of the application of this function, see Fig. SM 13b.

a

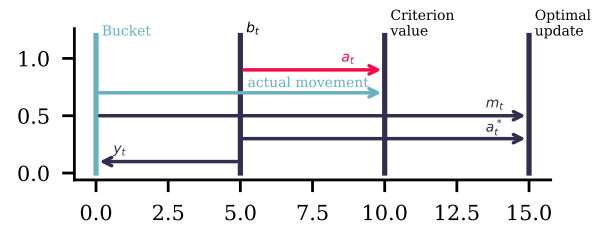

$\mathrm{b}$

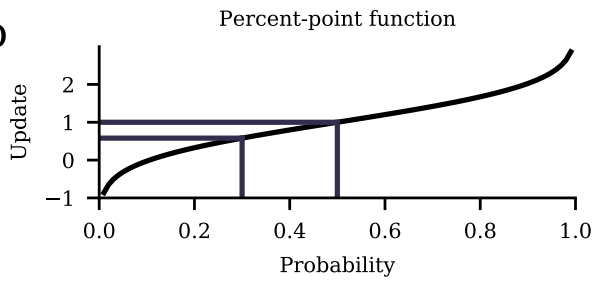

Stable- vs. shifting-bucket

C

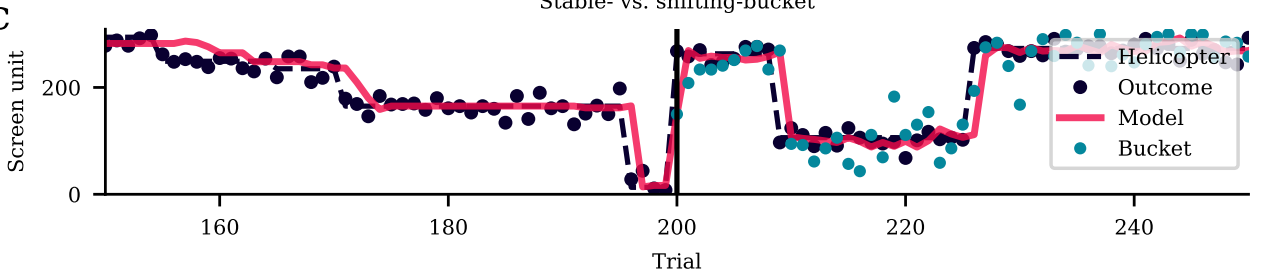

$\mathrm{d}$

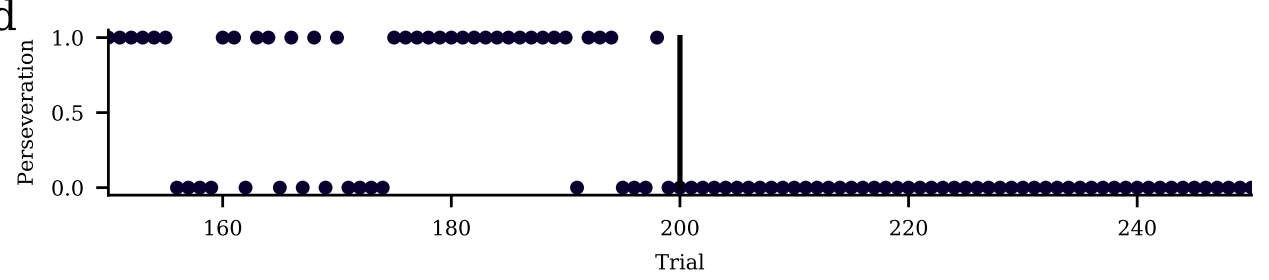

Figure SM 13. Satisficing model. a) For the satisficing model, we considered several variables. $y_{t}$ denotes the bucket shift. $a_{t}^{*}$ denotes the optimal belief update according to the reduced Bayesian model. $m_{t}$ denotes the spatial bucket movement that would be required to indicate the optimal update. In the example, this corresponds to $m_{t}=-y_{t}+a_{t}^{*}=-(-5)+10=15$. $a_{t}$ reflects the actual belief update (here in non-perseveration trials), which stops at the criterion value $w_{t}$. In the example this corresponds to $a_{t}=y_{t}+w_{t}=(-5)+10=5$. Finally, "actual movement" reflects the combination of $a_{t}$ and $y_{t}$, i.e., $-y_{t}+a_{t}=-(-5)+5=10$. b) Illustration of the percent-point function. The update that is less than or equal to the criterion of $p=0.3$, is approximately 0.58 , and for the criterion $p=0.5$, we have an update of approximately 1 . c) The plot shows model behavior in the stable- and shifting-bucket environment. d) The model frequently perseverated in the stable-bucket environment, but not in the shifting-bucket environment. 


\section{Identical Picture and Spot-a-Word tests}
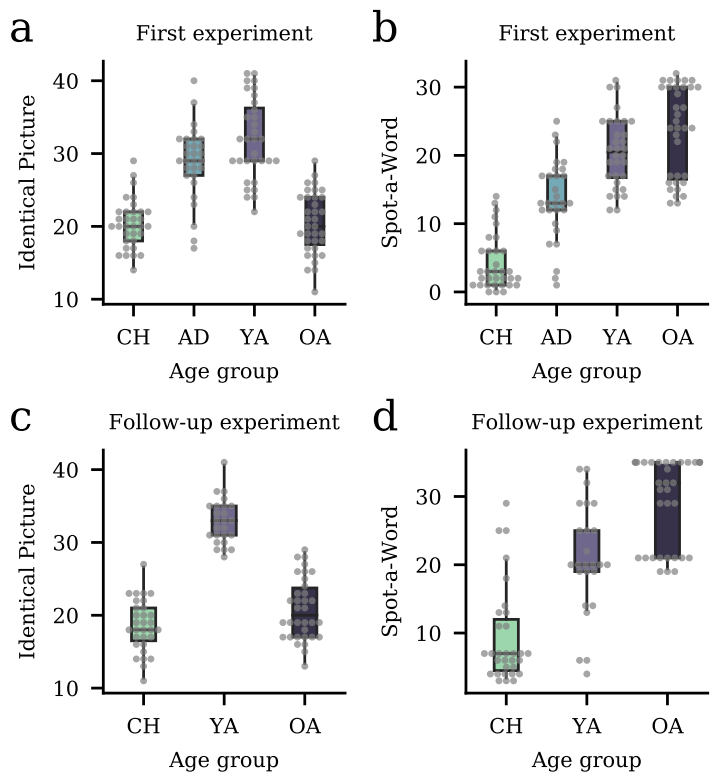

Figure SM 14. Processing speed and verbal knowledge across the two experiments. a) Identical Picture test first experiment. b) Spot-a-Word test first experiment. c) Identical Picture test follow-up experiment. d) Spot-a-Word test follow-up experiment.

Across both experiments, participants completed the Identical Picture test measuring processing speed and the Spot-a-Word test measuring verbal knowledge (Lindenberger et al., 1993). As detailed below, our results are in line with previous studies showing age-related declines in fluid abilities and age-related increases in crystallized abilities (Li et al., 2004).

In the first experiment (Fig. SM 14a), an ANOVA showed significantly different Identical Picture scores between the age groups $(H=74.034, p<0.001)$. Children (median $=20$, IQR 18.0 to 22.0$)$ had significantly lower scores compared to adolescents (median $=29$, IQR 27.0 to $32.0 ; u=90.0, p<0.001$ ) and younger adults (median $=32$, IQR 29.0 to $36.25 ; u=31.0, p<0.001$ ) but not compared to older adults (median $=20$, IQR 17.5 to $24.0 ; u=564.0, p=0.873$ ). Adolescents had significantly higher scores compared with older adults $(u=914.0, p<0.001)$ but not compared to younger adults $(u=336.0, p=$ 0.064). Finally, younger adults had significantly higher scores compared to older adults $(u=1068.0, p<$ $0.001)$.

Regarding the Spot-a-Word test in the first experiment (Fig. SM 14b), an ANOVA yielded significant differences across the age groups $(H=82.768, p<0.001)$. Scores of children (median $=3.0$, IQR 1.0 to 6.0) were significantly lower compared to adolescents (median $=13.0$, IQR 12.0 to $17.0 ; u=102.5, p<$ 0.001 ), younger adults (median $=20.5$, IQR 16.75 to $25.0 ; u=6.5, p<0.001$ ), and older adults (median $=24.0$, IQR 16.5 to $30.0 ; u=4.0, p<0.001)$. Adolescents had significantly lower scores compared to younger adults $(u=176.0, p<0.001)$ and older adults $(u=147.5, p<0.001)$. Finally, the test yielded a significant difference between younger and older adults $(u=403.0, p=0.049)$.

In the follow-up experiment (Fig. SM 14c), an ANOVA indicated significantly different Identical Picture scores $(H=55.247, p<0.001)$. Children (median $=18$, IQR 16.5 to 21.0 ) had significantly lower scores compared to younger adults (median $=33$, IQR 31.0 to $35.0 ; u=0.0, p<0.001$ ) but not compared to older adults (median $=20$, IQR 17.0 to $23.75 ; u=382.0, p=0.057$ ). Finally, younger adults had significantly higher scores than older adults $(u=846.5, p<0.001)$. 
Concerning the Spot-a-Word test in the follow-up experiment (Fig. SM 14d), the age groups had significantly different scores as well $(H=50.842, p<0.001)$. Children's scores (median $=7$, IQR 4.5 to 12.0) were significantly lower compared to younger adults (median $=20$, IQR 19.0 to $25.0 ; u=128.0, p$ $<0.001$ ) and compared to older adults (median $=32$, IQR 21.0 to $35.0 ; u=41.5, p<0.001$ ). Finally, younger adults had significantly lower scores compared to older adults $(u=160.5, p<0.001)$. 


\section{Optimal and approximate Bayesian inference}

\section{Optimal inference}

Optimal Bayesian inference over the mean $\mu_{t}$ of the outcome-generating Gaussian distribution conditional on the observed outcomes $x_{1: t}$ can be performed by considering the conditional distribution

$$
p\left(\mu_{t} \mid x_{1: t}\right)=\frac{p\left(\mu_{t}, x_{1: t}\right)}{p\left(x_{1: t}\right)}
$$

where the joint distribution of $\mu_{t}$ and $x_{1: t}$ is the result of the following derivation:

$$
\begin{aligned}
p\left(\mu_{t}, x_{1: t}\right) & =\sum_{c_{t}} \int p\left(x_{1: t}, \mu_{t}, \mu_{t-1}, c_{t}\right) d \mu_{t-1} \\
& =\sum_{c_{t}} \int p\left(x_{t} \mid x_{1: t-1}, \mu_{t}, \mu_{t-1}, c_{t}\right) p\left(x_{1: t-1}, \mu_{t}, \mu_{t-1}, c_{t}\right) d \mu_{t-1} \\
& =\sum_{c_{t}} \int p\left(x_{t} \mid \mu_{t}\right) p\left(x_{1: t-1}, \mu_{t}, \mu_{t-1}, c_{t}\right) d \mu_{t-1} \\
& =\sum_{c_{t}} \int p\left(x_{t} \mid \mu_{t}\right) p\left(\mu_{t} \mid x_{1: t-1}, \mu_{t-1}, c_{t}\right) p\left(x_{1: t-1}, \mu_{t-1}, c_{t}\right) d \mu_{t-1} \\
& =\sum_{c_{t}} \int p\left(x_{t} \mid \mu_{t}\right) p\left(\mu_{t} \mid \mu_{t-1}, c_{t}\right) p\left(x_{1: t-1}, \mu_{t-1}, c_{t}\right) d \mu_{t-1} \\
& =\sum_{c_{t}} \int p\left(x_{t} \mid \mu_{t}\right) p\left(\mu_{t} \mid \mu_{t-1}, c_{t}\right) p\left(\mu_{t-1} \mid x_{1: t-1}, c_{t}\right) p\left(x_{1: t-1}, c_{t}\right) d \mu_{t-1} \\
& =\sum_{c_{t}} \int p\left(x_{t} \mid \mu_{t}\right) p\left(\mu_{t} \mid \mu_{t-1}, c_{t}\right) p\left(\mu_{t-1} \mid x_{1: t-1}\right) p\left(x_{1: t-1}, c_{t}\right) d \mu_{t-1} \\
& =\sum_{c_{t}} \int p\left(x_{t} \mid \mu_{t}\right) p\left(\mu_{t} \mid \mu_{t-1}, c_{t}\right) p\left(\mu_{t-1} \mid x_{1: t-1}\right) p\left(x_{1: t-1} \mid c_{t}\right) p\left(c_{t}\right) d \mu_{t-1} \\
& =\sum_{c_{t}} \int p\left(x_{t} \mid \mu_{t}\right) p\left(\mu_{t} \mid \mu_{t-1}, c_{t}\right) p\left(\mu_{t-1} \mid x_{1: t-1}\right) p\left(c_{t}\right) d \mu_{t-1}
\end{aligned}
$$

and the marginal distribution over outcomes $x_{1: t}$ can be obtained by the integral

$$
p\left(x_{1: t}\right)=\int p\left(\mu_{t}, x_{1: t}\right) d \mu_{t}
$$

which together thus yields

$$
p\left(\mu_{t} \mid x_{1: t}\right)=\frac{\sum_{c_{t}} \int p\left(x_{t} \mid \mu_{t}\right) p\left(\mu_{t} \mid \mu_{t-1}, c_{t}\right) p\left(\mu_{t-1} \mid x_{1: t-1}\right) p\left(c_{t}\right) d \mu_{t-1}}{\int p\left(\mu_{t}, x_{1: t}\right) d \mu_{t}} .
$$

Expanding the sum in the numerator, i.e.,

$$
\begin{aligned}
& p\left(\mu_{t} \mid x_{1: t}\right)= \\
& \frac{\int p\left(x_{t} \mid \mu_{t}\right) p\left(\mu_{t} \mid \mu_{t-1}, c_{t}=0\right) p\left(\mu_{t-1} \mid x_{1: t-1}\right) p\left(c_{t}=0\right) d \mu_{t-1}+\int p\left(x_{t} \mid \mu_{t}\right) p\left(\mu_{t} \mid \mu_{t-1}, c_{t}=1\right) p\left(\mu_{t-1} \mid x_{1: t-1}\right) p\left(c_{t}=1\right) d \mu_{t-1}}{\int p\left(\mu_{t}, x_{1: t}\right) d \mu_{t}}
\end{aligned}
$$


shows that this inference scheme considers the case that a changepoint did not occur (first term) and that a changepoint occurred (second term). To keep track of this mixture distribution over many trials, messagepassing algorithms that consider all possible mixtures between changepoint and no-changepoint trials have been applied in previous work (Adams and MacKay, 2007; Wilson et al., 2010). These methods, however, require a wealth of computations, which arguably go beyond human-level capacities (Nassar et al., 2010). Therefore, Nassar and colleagues proposed a reduced Bayesian model that requires fewer computations and shares the core assumptions of the optimal Bayesian solution.

\section{Approximate inference}

The reduced Bayesian model approximates the mixture over all combinations of no-changepoint and changepoint trials with a Gaussian distribution that has a similar mean and variance as the mixture distribution computed in optimal Bayesian inference. Notably, the reduced model also considers the scenario of "no changepoint" and "changepoint"; however, it only concerns the current trial $t$ instead of all trials $1: t$.

We first show the derivation of the mixture component that assumes the absence of a changepoint. In the absence of changepoints, one can sequentially update a conjugate Gaussian distribution over the mean of the outcome-generating Gaussian distribution according to

$$
\begin{aligned}
\mu_{t+1} & =\frac{\sigma^{2}}{\sigma^{2}+\sigma_{t}^{2}} \mu_{t}+\frac{\sigma_{t}^{2}}{\sigma^{2}+\sigma_{t}^{2}} x_{t} \\
& =\left(\left(1-\tau_{t}\right) \mu_{t}+\tau_{t} x_{t}\right) \\
& =\mu_{t}+\tau_{t} x_{t}-\tau \mu_{t} \\
& =\mu_{t}+\tau_{t}\left(x_{t}-\mu_{t}\right)
\end{aligned}
$$

where

$$
\tau_{t}:=\frac{\sigma_{t}^{2}}{\sigma^{2}+\sigma_{t}^{2}}
$$

refers to the relative uncertainty over the estimated Gaussian mean parameter. Relative uncertainty can be updated according to

$$
\tau_{t+1}=\tau_{t} \sigma^{2}
$$

Next, we consider the case that a changepoint occurred. Recall that a changepoint renders the past irrelevant, and therefore, under the assumption of an uninformative prior expectation over the parameter, the best prediction is most the recent outcome $x_{t}$.

To appropriately weight these two mixture components, the reduced Bayesian model computes changepoint probability $\omega_{t}$, defined as

$$
\omega_{t}:=p\left(c_{t}=1 \mid x_{t}\right)=\frac{p\left(c_{t}=1, x_{t}\right)}{p\left(x_{t}\right)},
$$

where

$$
p\left(x_{t}\right)=\sum_{c_{t}} p\left(x_{t} \mid c_{t}\right) p^{h}\left(c_{t}\right)
$$


and thus

$$
\begin{aligned}
p\left(c_{t}=1 \mid x_{t}\right) & =\frac{p\left(x_{t} \mid c_{t}=1\right) p^{h}\left(c_{t}=1\right)}{\sum_{c_{t}} p\left(x_{t} \mid c_{t}\right) p^{h}\left(c_{t}\right)} \\
& =\frac{p\left(x_{t} \mid c_{t}=1\right) p^{h}\left(c_{t}=1\right)}{p\left(x_{t} \mid c_{t}=0\right) p^{h}\left(c_{t}=0\right)+p\left(x_{t} \mid c_{t}=1\right) p^{h}\left(c_{t}=1\right)} \\
& =\frac{U\left(\delta_{t} ;[0,300]\right) h}{N\left(\delta_{t} ; 0, \sigma_{t}^{2}+\sigma^{2}\right)(1-h)+U\left(\delta_{t} ;[0,300]\right)} .
\end{aligned}
$$

The reduced Bayesian model then computes the mean of the outcome-generating distribution as a function of the two mixture components weighted by changepoint probability

$$
\mu_{t+1}=\left(\mu_{t+1} \mid c_{t}=0\right)\left(1-\omega_{t}\right)+\left(\mu_{t+1} \mid c_{t}=1\right) \omega_{t}
$$

which can be reformulated to an error-driven learning rule as follows

$$
\begin{aligned}
\mu_{t+1} & =\left(\mu_{t+1} \mid c_{t}=0\right)\left(1-\omega_{t}\right)+\left(\mu_{t+1} \mid c_{t}=1\right) \omega_{t} \\
& =\left(\left(1-\tau_{t}\right) \mu_{t}+\tau_{t} x_{t}\right)\left(1-\omega_{t}\right)+x_{t} \omega_{t} \\
& =\left(\mu_{t}+\tau_{t} x_{t}-\tau \mu_{t}\right)\left(1-\omega_{t}\right)+x_{t} \omega_{t} \\
& =\left(\mu_{t}+\tau_{t}\left(x_{t}-\mu_{t}\right)\right)\left(1-\omega_{t}\right)+x_{t} \omega_{t} \\
& =\left(\mu_{t}+\tau_{t}\left(x_{t}-\mu_{t}\right)\right)\left(1-\omega_{t}\right)+\left(\mu_{t}+\left(x_{t}-\mu_{t}\right)\right) \omega_{t} \\
& =\left(\mu_{t}+\tau_{t} \delta_{t}\right)\left(1-\omega_{t}\right)+\left(\mu_{t}+\delta_{t}\right) \omega_{t} \\
& =\mu_{t}-\mu_{t} \omega_{t}+\tau_{t} \delta_{t}-\tau_{t} \delta_{t} \omega_{t}+\mu_{t} \omega_{t}+\delta_{t} \omega_{t} \\
& =\mu_{t}-\mu_{t} \omega_{t}+\mu_{t} \omega_{t}+\tau_{t} \delta_{t}-\tau_{t} \delta_{t} \omega_{t}+\delta_{t} \omega_{t} \\
& =\mu_{t}+\tau_{t} \delta_{t}-\tau_{t} \delta_{t} \omega_{t}+\delta_{t} \omega_{t} \\
& =\mu_{t}+\left(\tau_{t}-\tau_{t} \omega_{t}+\omega_{t}\right) \delta_{t} \\
& =\mu_{t}+\left(\tau_{t}+\omega_{t}-\tau_{t} \omega_{t}\right) \delta_{t} \\
& =\mu_{t}+\alpha_{t} \delta_{t}
\end{aligned}
$$

where

$$
\alpha_{t}:=\tau_{t}+\omega_{t}-\tau_{t} \omega_{t}
$$

Finally, estimation uncertainty, that is, the variance of the approximated mixture distribution $\sigma_{t+1}^{2}$, is the $\omega_{t}$-weighted mixture of the no-changepoint and changepoint component

$$
\sigma_{t+1}^{2}:=\omega_{t} \sigma^{2}+\left(1-\omega_{t}\right) \tau_{t} \sigma^{2}+\omega_{t}\left(1-\omega_{t}\right)\left(\delta_{t}\left(1-\tau_{t}\right)\right)^{2},
$$

which follows from substituting the conditional means and variances into the equation of the variance of a mixture of two Gaussian distributions

$$
\begin{aligned}
\sigma_{t+1}^{2} & =\left(\sigma_{t+1}^{2} \mid c_{t}=0\right)\left(1-\omega_{t}\right)+\left(\sigma_{t+1}^{2} \mid c_{t}=1\right) \omega_{t}+\left(1-\omega_{t}\right) \omega_{t}\left(\left(\mu_{t+1} \mid c_{t}=0\right)-\left(\mu_{t+1} \mid c_{t}=1\right)\right)^{2} \\
& =\tau_{t} \sigma^{2}\left(1-\omega_{t}\right)+\sigma^{2} \omega_{t}+\omega_{t}\left(1-\omega_{t}\right)\left(\left(\mu_{t}+\tau_{t} \delta_{t}\right)-\left(\mu_{t}+\delta_{t}\right)\right)^{2} \\
& =\tau_{t} \sigma^{2}\left(1-\omega_{t}\right)+\sigma^{2} \omega_{t}+\omega_{t}\left(1-\omega_{t}\right)\left(\tau_{t} \delta_{t}-\delta_{t}\right)^{2} \\
& =\tau_{t} \sigma^{2}\left(1-\omega_{t}\right)+\sigma^{2} \omega_{t}+\omega_{t}\left(1-\omega_{t}\right)\left(\delta_{t}\left(\tau_{t}-1\right)\right)^{2} \\
& =\omega_{t} \sigma^{2}+\left(1-\omega_{t}\right) \tau_{t} \sigma^{2}+\omega_{t}\left(1-\omega_{t}\right)\left(\delta_{t}\left(1-\tau_{t}\right)\right)^{2}
\end{aligned}
$$




\section{Catch-trial derivations}

During catch trials, we assumed that the provided helicopter cue that was centered on the true mean was perceived as an ambiguous cue that corresponded to a Gaussian probability density function (PDF) where the mean corresponded to the true helicopter location and the variance that expressed the reliability of the cue was assumed to be different between participants. In effect, the updated mean and variance followed from the multiplication of the Gaussian PDF over the learned helicopter location based on the history of outcomes and the Gaussian PDF over the presented helicopter position, where the covariance between the two distribution corresponds to

$$
C:=\frac{1}{\frac{1}{\sigma_{t+1}^{2}}+\frac{1}{\sigma_{H}^{2}}}
$$


and the mean corresponds to

$$
\begin{aligned}
& \mu_{t+1}=C \frac{1}{\sigma_{t+1}^{2}} \mu_{t+1}+C \frac{1}{\sigma_{H}^{2}} \mu_{H} \\
& =\frac{1}{\frac{1}{\sigma_{t+1}^{2}}+\frac{1}{\sigma_{H}^{2}}} \frac{1}{\sigma_{t+1}^{2}} \mu_{t+1}+\frac{1}{\frac{1}{\sigma_{t+1}^{2}}+\frac{1}{\sigma_{H}^{2}}} \frac{1}{\sigma_{H}^{2}} \mu_{H} \\
& =\frac{1}{\sigma_{t+1}^{2}\left(\frac{1}{\sigma_{t+1}^{2}}+\frac{1}{\sigma_{H}^{2}}\right)} \mu_{t+1}+\frac{1}{\frac{1}{\sigma_{t+1}^{2}}+\frac{1}{\sigma_{H}^{2}}} \frac{1}{\sigma_{H}^{2}} \mu_{H} \\
& =\frac{1}{\sigma_{t+1}^{2} \frac{\sigma_{H}^{2}}{\sigma_{t+1}^{2} \sigma_{H}^{2}}+\frac{\sigma_{t+1}^{2}}{\sigma_{t+1}^{2} \sigma_{H}^{2}}} \mu_{t+1}+\frac{1}{\frac{1}{\sigma_{t+1}^{2}}+\frac{1}{\sigma_{H}^{2}}} \frac{1}{\sigma_{H}^{2}} \mu_{H} \\
& =\frac{1}{\sigma_{t+1}^{2} \frac{\sigma_{H}^{2}+\sigma_{t+1}^{2}}{\sigma_{t+1}^{2} \sigma_{H}^{2}}} \mu_{t+1}+\frac{1}{\frac{1}{\sigma_{t+1}^{2}}+\frac{1}{\sigma_{H}^{2}}} \frac{1}{\sigma_{H}^{2}} \mu_{H} \\
& =\frac{\sigma_{t+1}^{2} \sigma_{H}^{2}}{\sigma_{t+1}^{2}\left(\sigma_{H}^{2}+\sigma_{t+1}^{2}\right)} \mu_{t+1}+\frac{1}{\frac{1}{\sigma_{t+1}^{2}}+\frac{1}{\sigma_{H}^{2}}} \frac{1}{\sigma_{H}^{2}} \mu_{H} \\
& =\frac{\sigma_{H}^{2}}{\sigma_{H}^{2}+\sigma_{t+1}^{2}} \mu_{t+1}+\frac{1}{\frac{1}{\sigma_{t+1}^{2}}+\frac{1}{\sigma_{H}^{2}}} \frac{1}{\sigma_{H}^{2}} \mu_{H} \\
& =\frac{\sigma_{H}^{2}}{\sigma_{H}^{2}+\sigma_{t+1}^{2}} \mu_{t+1}+\frac{1}{\sigma_{H}^{2}\left(\frac{1}{\sigma_{t+1}^{2}}+\frac{1}{\sigma_{H}^{2}}\right)} \mu_{H} \\
& =\frac{\sigma_{H}^{2}}{\sigma_{H}^{2}+\sigma_{t+1}^{2}} \mu_{t+1}+\frac{1}{\sigma_{H}^{2} \frac{\sigma_{H}^{2}}{\sigma_{t+1}^{2} \sigma_{H}^{2}}+\frac{\sigma_{t+1}^{2}}{\sigma_{t+1}^{2} \sigma_{H}^{2}}} \mu_{H} \\
& =\frac{\sigma_{H}^{2}}{\sigma_{H}^{2}+\sigma_{t+1}^{2}} \mu_{t+1}+\frac{1}{\sigma_{H}^{2} \frac{\sigma_{H}^{2}+\sigma_{t+1}^{2}}{\sigma_{t+1}^{2} \sigma_{H}^{2}}} \mu_{H} \\
& =\frac{\sigma_{H}^{2}}{\sigma_{H}^{2}+\sigma_{t+1}^{2}} \mu_{t+1}+\frac{\sigma_{t+1}^{2} \sigma_{H}^{2}}{\sigma_{H}^{2}\left(\sigma_{t+1}^{2}+\sigma_{H}^{2}\right)} \mu_{H} \\
& =\frac{\sigma_{H}^{2}}{\sigma_{H}^{2}+\sigma_{t+1}^{2}} \mu_{t+1}+\frac{\sigma_{t+1}^{2}}{\sigma_{t+1}^{2}+\sigma_{H}^{2}} \mu_{H} \\
& =\left(1-\frac{\sigma_{t+1}^{2}}{\sigma_{t+1}^{2}+\sigma_{H}^{2}}\right) \mu_{t+1}+\frac{\sigma_{t+1}^{2}}{\sigma_{t+1}^{2}+\sigma_{H}^{2}} \mu_{H} \\
& =(1-w) \mu_{t+1}+w \mu_{H}
\end{aligned}
$$

where

$$
w:=\frac{\sigma_{t+1}^{2}}{\sigma_{t+1}^{2}+\sigma_{H}^{2}} .
$$

